Boise State University

ScholarWorks

4-4-2008

\title{
Raman Spectroscopy of Ferroelectric Thin Films and Superlattices
}

Dmitri Tenne

Boise State University

Xiaoxing Xi

Pennsylvania State University 
Published in Journal of the American Ceramic Society, Vol. 91, pp. 1820 -1834 (2008).

\title{
Raman spectroscopy of ferroelectric thin films and superlattices*
}

\author{
Dmitri A. Tenne ${ }^{\dagger}$ \\ Department of Physics, Boise State University, Boise, ID 83725-1570 \\ Xiaoxing $\mathrm{Xi}$ \\ Department of Physics, Department of Materials Science \\ and Engineering, and Materials Research Institute, \\ the Pennsylvania State University, University Park, PA 16802
}

(Dated: January 16, 2008)

\begin{abstract}
Recent results of Raman spectroscopy studies of lattice dynamics and phase transitions in ferroelectric thin films and superlattices are reviewed. Raman studies of $\mathrm{SrTiO}_{3}, \mathrm{BaTiO}_{3}$, and $\mathrm{Ba}_{x} \mathrm{Sr}_{1-x} \mathrm{TiO}_{3}$ thin films in comparison to corresponding single crystals are presented; essential differences in the lattice dynamics behavior of thin films and single crystals are discussed. Application of ultraviolet Raman spectroscopy for studies of nanoscale ferroelectric heterostructures, such as $\mathrm{BaTiO}_{3} / \mathrm{SrTiO}_{3}$ superlattices, is demonstrated.
\end{abstract}


This is an author-produced, peer-reviewed version of this article. The final, definitive version of this document can be found online at Journal of the

American Ceramic Society, published by Blackwell Publishing. Copyright restrictions may apply. doi: 10.1111/j.1551-2916.2008.02371.x

\section{INTRODUCTION}

In the past 15 years, thin films and heterostructures based on perovskite oxide ferroelectrics have been extensively studied for wide variety of device applications such as non-volatile ferroelectric memories, dynamic random access memories or tunable microwave devices. ${ }^{1-4}$ The difference in the properties of thin films compared to those of corresponding bulk materials has been a major issue. For example, while the bulk ferroelectrics such as $\mathrm{Ba}_{x} \mathrm{Sr}_{1-x} \mathrm{TiO}_{3}$ show sharp peaks reaching the values of $10^{4}$ in the temperature dependence of the dielectric constant $\varepsilon,{ }^{14}$ thin films of the same material exhibit a broad maximum with the peak values an order of magnitude smaller, and a larger dielectric loss. ${ }^{4,5}$

Recent advances in epitaxial technology of oxide materials ${ }^{6}$ have opened a new stage in the field, making possible an experimental realization of complex artificial oxide nanoscale heterostructures with atomic-level thickness control comparable to that developed for semiconductor heterostructures. High quality ultrathin films of ferroelectric materials can be grown and combined with other ferroelectrics and such materials as superconducting and magnetic oxides, semiconductors, allowing fabrication of multifunctional structures and opening exciting opportunities for potential device applications. Reduction of the structural dimensions to the nanometer scale gives rise to new physical phenomena and properties dramatically different from those of homogeneous bulk ferroelectrics ${ }^{3,7,8}$. The continuous demand for device miniaturization requires the behavior of ferroelectric nanoscale films and heterostructures to be understood.

Of central importance for understanding the fundamental properties of ferroelectrics is dynamics of crystal lattice. ${ }^{9}$ The soft-mode theory of ferroelectricity ${ }^{10}$ has established the relationship between the polar optical vibrational modes and ferroelectric polarization. The lowest-frequency transverse optical phonon, the soft mode, involves the same atomic displacements as those responsible for the appearance of spontaneous polarization. When the temperature approaches the Curie temperature $T_{c}$, the soft-mode frequency tends to zero, becoming frozen in the crystal, which transforms to the ferroelectric phase. ${ }^{10}$, thus the soft mode instability at $T_{c}$ causes the ferroelectric phase transition. The soft mode behavior is related to such properties of ferroelectric materials as high dielectric constant, large piezoelectric coefficients, dielectric nonlinearity, which are extremely important for technological applications. Lyddane-Sachs-Teller (LST) relation connects the macroscopic dielectric con- 
This is an author-produced, peer-reviewed version of this article. The final, definitive version of this document can be found online at Journal of the

stants of a material with its microscopic properties - optical phonon frequencies:

$$
\varepsilon_{0}=\varepsilon_{\infty} \prod \frac{\omega_{L O_{j}}^{2}}{\omega_{T O_{j}}^{2}}
$$

where $\varepsilon_{0}$ and $\varepsilon_{\infty}$ are the static and high-frequency dielectric constants, $\omega_{L O}$ and $\omega_{T O}$ ) are the frequencies of longitudinal and transverse optical phonons, respectively. Usually the frequencies of higher optical phonons vary slightly with temperature, and the temperature dependence of $\varepsilon_{0}$ is mostly determined by the behavior of the soft mode. The decrease of the soft mode frequency as the temperature approaches to $T_{c}$ causes a dramatic increase of the dielectric constant. The soft-mode behavior is also responsible for the dielectric nonlinearity, i. e. the electric-field dependence of $\varepsilon_{0}$. The electric field-induced hardening, i.e. the increase of the soft mode frequency $\omega_{T O}$, according to the LST relation, leads to the decrease of $\varepsilon_{0}$. Another important materials parameter for applications, the dielectric loss, is also related to the soft mode behavior. In an ideal ferroelectric crystal, it is determined by the damping of the soft mode through multiple-phonon processes in the paraelectric phase, and dominated by the quasi-Debye contribution in the ferroelectric phase. ${ }^{11-13}$.

Lattice dynamics in bulk perovskite oxide ferroelectrics has been investigated for several decades using neutron scattering, ${ }^{15-20}$, far infrared spectroscopy,${ }^{21-23}$ and Raman scattering. Raman spectroscopy is one of the most powerful analytical techniques for studying the lattice vibrations and other elementary excitations in materials providing important information about the structure, composition, strain, defects, phase transitions. This technique was successfully applied to bulk perovskite oxides, such as barium titanate, $\mathrm{BaTiO}_{3},{ }^{24-31}$ strontium titanate, $\mathrm{SrTiO}_{3},{ }^{32-34}$ lead titanate, $\mathrm{PbTiO}_{3} .{ }^{35}$

Study of lattice dynamics in ferroelectric thin films and nanostructures is a challenging task. A difficulty in applying conventional Raman spectroscopy for thin films of ferroelectrics and other wide-bandgap materials lies in the fact that the visible photon energy is much smaller than the bandgap. Consequently, the absorption of light is extremely weak and the penetration depth is large, allowing light to travel through thin film into the much thicker substrate. Much larger scattering volume of the substrate thus generates overwhelming signals in the Raman spectra. Several approaches were used to overcome this difficulty. In previous Raman studies of ferroelectric thin films, the films were grown either on reflective substrates, such as $\mathrm{Pt} / \mathrm{Si},{ }^{36}$ or on substrates with low Raman activity at the frequency of 
This is an author-produced, peer-reviewed version of this article. The final, definitive version of this document can be found online at Journal of the American Ceramic Society, published by Blackwell Publishing. Copyright restrictions may apply. doi: 10.1111/j.1551-2916.2008.02371.x

interest, such as $\mathrm{Al}_{2} \mathrm{O}_{3},{ }^{37,38} \mathrm{KTaO}_{3},{ }^{39}$, fused silica, ${ }^{40}$, and $\mathrm{MgO} .{ }^{40-44}$ But for many applications these are not the substrates of choice in terms of growth of high-quality defect-free epitaxial films and structures because of lattice and thermal expansion mismatch problems, and the crystalline quality of the ferroelectric thin films is often compromised. In our studies of relatively thick (over $100 \mathrm{~nm}$ ) $\mathrm{SrTiO}_{3}, \mathrm{BaTiO}_{3}, \mathrm{Ba}_{x} \mathrm{Sr}_{1-x} \mathrm{TiO}_{3}$ films we employed the metal-oxide bilayer technique using an opaque layer of a conductive oxide, such as $\mathrm{SrRuO}_{3}$ to eliminate the substrate contribution. ${ }^{45-51}$ In contrast to thin film growth on substrates like $\mathrm{Pt} / \mathrm{Si}$ or $\mathrm{Al}_{2} \mathrm{O}_{3}$, the metaloxide layer allows the growth of the epitaxial ferroelectric thin films with significantly higher structural quality. This technique was also successfully applied for study of the soft modes in $\mathrm{SrTiO}_{3}$ films by far infrared ellipsometry. ${ }^{52}$

However, for ultrathin films (less than $100 \mathrm{~nm}$ ) and nanostructures, another approach is required. In such thin films, the intensity of Raman scattering using conventional (visible) excitation sources is extremely low, and even weak signal from the conductive oxide layer used to block the substrate can prevent the observation of the phonons of the nanometerthick film. We have recently demonstrated that Raman spectroscopy using an ultraviolet (UV) excitation can be successfully applied for studies of nanoscale ferroelectrics. ${ }^{53}$ In the present paper we summarize our recent results on Raman spectroscopy of thin films of $\mathrm{SrTiO}_{3}, \mathrm{BaTiO}_{3}, \mathrm{Ba}_{x} \mathrm{Sr}_{1-x} \mathrm{TiO}_{3}$, obtained using the metaloxide bilayer technique, and first UV Raman results on $\mathrm{BaTiO}_{3} / \mathrm{SrTiO}_{3}$ periodic multilayer structures - superlattices.

\section{PHONONS IN BULK BATIO ${ }_{3}$, SRTIO $_{3}$ AND BA SR $_{1-x}$ TIO $_{3}$}

Bulk $\mathrm{BaTiO}_{3}, \mathrm{SrTiO}_{3}$ and their solid solution $\mathrm{Ba}_{x} \mathrm{Sr}_{1-x} \mathrm{TiO}_{3}$ have the same cubic perovskite structure in the high-temperature phase. The pure compounds, $\mathrm{BaTiO}_{3}$ and $\mathrm{SrTiO}_{3}$ exhibit different behaviors at lower temperatures. $\mathrm{BaTiO}_{3}$ undergoes 3 consecutive phase transitions from a cubic $m 3 m$ to a tetragonal $4 \mathrm{~mm}$ phase at $403 \mathrm{~K}$, then to an orthorhombic mm2 phase at $278 \mathrm{~K}$, and, finally, to a rhombohedral $3 m$ phase at $183 \mathrm{~K} .{ }^{14}$ The three lowtemperature phases are ferroelectric. In bulk $\mathrm{Ba}_{x} \mathrm{Sr}_{1-x} \mathrm{TiO}_{3}$ of Ba-rich compositions these three ferroelectric phases also exist, but the temperatures of three phase transitions decrease nearly linearly with decreasing $x$ and coincide at about $100 \mathrm{~K}$ and $x \approx 0.15 .{ }^{54}$ For lower Ba concentrations only one ferroelectric phase exists, which disappears at $x<0.035 .{ }^{54}$ Bulk $\mathrm{SrTiO}_{3}$ is an incipient ferroelectric (quantum paraelectric), in which the ferroelectric phase 
This is an author-produced, peer-reviewed version of this article. The final, definitive version of this document can be found online at Journal of the American Ceramic Society, published by Blackwell Publishing. Copyright restrictions may apply. doi: 10.1111/j.1551-2916.2008.02371.x

transition is suppressed by quantum fluctuations. ${ }^{55}$ An antiferrodistortive cubic-tetragonal phase transition occurs in $\mathrm{SrTiO}_{3}$ at $105 \mathrm{~K}$, which involves the rotation of the Ti-O octahedra. The resulting tetragonal structure $4 / \mathrm{mmm}$ is still centrosymmetric and, hence, not ferroelectric. The transition to the ferroelectric phase can be induced by doping, ${ }^{56,57}$ isotope substitution, ${ }^{58}$ or stress. ${ }^{59-61}$

The perovskite structure has 5 atoms per unit cell, therefore, there are 12 optical vibrational modes. In the cubic phase $m 3 m$ the zone center optical phonons belong to $3 F_{1 u}+F_{2 u}$ irreducible representations. Each of the $F_{u}$ modes is triply degenerate, and all of them are of odd parity with respect to the inversion, therefore, forbidden in the first order Raman scattering. The $F_{1 u}$ modes are infrared active, while the $F_{2 u}$ modes are silent. When the temperature decreases, $\mathrm{Ba}_{x} \mathrm{Sr}_{1-x} \mathrm{TiO}_{3}$ becomes ferroelectric for $x \geq 0.035,{ }^{54}$ thus breaking the inversion symmetry, and the Raman-active modes appear. Upon transition from the cubic to the tetragonal phase the $F_{1 u}$ modes split into $A_{1}$ and $E$ modes, and the $F_{2 u}$ phonons gives rise to $B_{1}$ and $E$ modes. The $E$ modes are doubly degenerate. In the orthorhombic phase the optical phonons belong to $A_{1}, A_{2}, B_{1}$, and $B_{2}$ symmetry. In the lowest-temperature, rhombohedral phase, the modes, originating from the cubic $F_{1 u}$ phonons are split into $A_{1}$ and $E$ modes, while the $F_{2 u}$ vibrations produce $A_{2}$ and $E$ modes. The symmetric $A_{1}$ and $E$ modes are Raman-active. For the $A_{1}$ phonons the atomic displacements are parallel to the $z$ axis (polar axis in the ferroelectric phases), while for the $E$ modes atoms vibrate in the $x y$ plane perpendicular to the polar axis. Also, in polar crystals, to which barium strontium titanate belongs, long-range electrostatic interaction results in additional splitting of each optical phonon branch into transverse (TO) and longitudinal (LO) modes.

In $\mathrm{BaTiO}_{3}$, the $F_{1 u}$ soft mode is heavily overdamped in the paraelectric cubic phase. ${ }^{25-27}$ (The term "overdamping" usually implies the ratio $\Gamma / \omega>\sqrt{2}$, where $\Gamma$ is the phonon mode linewidth, and $\omega$ is the mode frequency. Upon this condition there is no distinct peak at finite frequency in Raman spectra.) In the tetragonal phase, the $E$ component of the soft mode remains overdamped..$^{25-27}$ In the orthorhombic phase, Laabidi et $a l .{ }^{62}$ reported the damping of the soft mode to decrease, so the mode becomes underdamped below $253 \mathrm{~K}$. At the transition to the rhombohedral phase, according to Laabidi et al. ${ }^{62}$ the soft mode abruptly stiffens up to $\sim 200 \mathrm{~cm}^{-1}$. This heavy overdamping of the soft mode in $\mathrm{BaTiO}_{3}$ has been attributed to the order-disorder character of the ferroelectric phase transition in this material. For a long time since the discovery of ferroelectricity, barium titanate had 
This is an author-produced, peer-reviewed version of this article. The final, definitive version of this document can be found online at Journal of the American Ceramic Society, published by Blackwell Publishing. Copyright restrictions may apply. doi: 10.1111/j.1551-2916.2008.02371.x

been considered as an example of displacive ferroelectrics, ${ }^{9}$ supported by earlier neutron, ${ }^{63}$ Raman ${ }^{25-27}$ and hyper-Raman ${ }^{28}$ experiments, which demonstrated the existence of the soft TO phonon mode and its softening in the paraelectric phase with decreasing the temperature. However, the soft mode becomes highly overdamped near the Curie temperature, and its frequency does not extrapolate to zero at $T_{c}$. A discrepancy was observed near $T_{c}$ between the dielectric constant obtained from capacitance measurements and from the soft mode frequencies via the LST relation.

The order-disorder picture was initially suggested for $\mathrm{BaTiO}_{3}$ in order to explain the observation of strong diffusive x-ray scattering. ${ }^{64}$ It was later supported by the observation of the symmetry-forbidden first-order Raman lines above $T_{c}$, in the cubic phase, and by electronic paramagnetic resonance (EPR) studies. ${ }^{65}$ An eight-site order-disorder model was proposed, in which the Ti ions in the paraelectric phase do not reside in the center of the cubic unit cell. Instead there are eight equivalent off-center energy minima located along [111] directions. In the high-temperature phase all sites are occupied by Ti ion with equal probability, and the average symmetry is cubic. Four sites become preferentially occupied in the tetragonal phase, only two nearest-neighbor sites are the most probably occupied in the orthorhombic phase, and only one site remains occupied in rhombohedral phase. The $a b$ initio calculations of the total energy as a function of Ti ion displacements ${ }^{66}$ in the cubic phase show the presence of absolute energy minima at the [111] off-center positions, while local (higher energy) minima exist for [100] displacements. The low-frequency $\left(\sim 10^{8} \mathrm{~Hz}\right)$ motion of $\mathrm{Ti}$ ion between equivalent off-center cites was suggested to be responsible for the discrepancy between the dielectric constant and soft mode behavior near the Curie temperature. This relaxational motion leads to the appearance of intensive low-frequency near-excitation scattering in Raman spectra (so called central peak). The observation of a central peak in the Raman spectra of $\mathrm{BaTiO}_{3}{ }^{67,68}$ provided the evidence of such a slow relaxational motion. Later femtosecond time-resolved stimulated Raman measurements ${ }^{69}$ allowed to distinguish between overdamped soft modes and relaxational modes.

Recent nuclear magnetic resonance studies ${ }^{70}$ demonstrated the coexistence of both displacive and order-disorder components in the phase transition mechanism in $\mathrm{BaTiO}_{3}$ and the breakdown of local cubic symmetry due to the motion of Ti ions between off-center sites in the paraelectric phase. Theoretical models were developed ${ }^{71-74}$ which describe the dynamics of ions in $\mathrm{BaTiO}_{3}$ as consisting of two components with different time scale: rapid 
This is an author-produced, peer-reviewed version of this article. The final, definitive version of this document can be found online at Journal of the

American Ceramic Society, published by Blackwell Publishing. Copyright restrictions may apply. doi: 10.1111/j.1551-2916.2008.02371.x

vibrations associated with the soft phonons and slow relaxational modes. In intermediate ferroelectrics like $\mathrm{BaTiO}_{3}$ there is strong coupling between the soft and relaxational modes, probably leading to overdamping of the soft mode. First-principles theory of ferroelectric phase transitions for $\mathrm{BaTiO}_{3}{ }^{75}$ also shows the phase transitions to be intermediate between order-disorder and displacive character.

In contrast to $\mathrm{BaTiO}_{3}, \mathrm{SrTiO}_{3}$ remains paraelectric at all temperatures, and the zonecenter optical phonons remain Raman inactive. The spectrum of $\mathrm{SrTiO}_{3}$ crystal is dominated by the second order features. Application of an electric field breaks the inversion symmetry, thus allowing the observation of the soft mode by Raman scattering. ${ }^{33}$ The soft mode is well-defined and does not tend to zero as temperature decreases, saturating at $\sim 32 \mathrm{~K}$ due to quantum fluctuations. The soft mode in $\mathrm{SrTiO}_{3}$ exhibits no overdamping over the entire temperature range, and excellent agreement is observed between the dielectric constant and the soft mode, both in temperature and electric field dependence. ${ }^{33}$ No significant disorder effects have been observed in $\mathrm{SrTiO}_{3}$, and therefore it is considered as predominantly displacive-type ferroelectric. Due to the antiferrodistortive phase transition at $105 \mathrm{~K}$, sharp peaks of $R$ modes, which are the zone-edge ( $R$ point) phonons, become Raman active via double folding of the Brillouin zone. ${ }^{32}$

As compared to pure $\mathrm{BaTiO}_{3}$ and $\mathrm{SrTiO}_{3}$, only few studies of lattice dynamics of bulk $\mathrm{Ba}_{x} \mathrm{Sr}_{1-x} \mathrm{TiO}_{3}$ were published, despite the large number of studies dealing with the properties of this technologically important material. For $\mathrm{Ba}_{x} \mathrm{Sr}_{1-x} \mathrm{TiO}_{3}$ ceramics the concentration dependence of phonon frequencies at the temperature of $6 \mathrm{~K}$ was reported ${ }^{76}$. Kuo et al. ${ }^{77}$ re- $^{\text {re }}$ ported combined Raman and X-ray diffraction studies of polycrystalline $\mathrm{Ba}_{x} \mathrm{Sr}_{1-x} \mathrm{TiO}_{3}$ samples in the entire composition range, but only at room temperature. Dobal et al. ${ }^{78}$ and Naik et $a l .{ }^{79}$ studied Raman spectra of bulk ceramic and thin-film polycrystalline $\mathrm{Ba}_{x} \mathrm{Sr}_{1-x} \mathrm{TiO}_{3}$ with large $x(x=0.65-1.0)$ at elevated temperatures focusing on the cubic-tetragonal phase transition temperature as a function of composition $x$. In these Ba-rich compositions, the authors observed broad first-order Raman features well beyond $T_{c}$, similar to the behavior of pure $\mathrm{BaTiO}_{3}$. The loss of intensity of several tetragonal-phase phonon modes was used to determine the ferroelectric to paraelectric phase-transition temperatures.

There has been lack of literature data on $\mathrm{Ba}_{x} \mathrm{Sr}_{1-x} \mathrm{TiO}_{3}$ single crystals, which is likely due to the difficulties in growing high purity crystals. We reported a detailed Raman study of $\mathrm{Ba}_{x} \mathrm{Sr}_{1-x} \mathrm{TiO}_{3}$ single crystals with different compositions $(x=0,0.05,0.1,0.2,0.35,0.5$, 
This is an author-produced, peer-reviewed version of this article. The final, definitive version of this document can be found online at Journal of the American Ceramic Society, published by Blackwell Publishing. Copyright restrictions may apply. doi: 10.1111/j.1551-2916.2008.02371.x

and 1) and measured between 5-300 K. ${ }^{80}$ The $\mathrm{Ba}_{x} \mathrm{Sr}_{1-x} \mathrm{TiO}_{3}$ single crystals for this study were grown by laser-heated pedestal growth technique. ${ }^{81}$ Addition of $\mathrm{Ba}$ to $\mathrm{SrTiO}_{3}$ changes the Raman spectra lattice dynamics significantly. Already for $x=0.05$ the crystal becomes ferroelectric at about $60 \mathrm{~K}$ and $T_{c}$ increases with $x$. Consequently, the low temperature Raman spectra of $\mathrm{Ba}_{x} \mathrm{Sr}_{1-x} \mathrm{TiO}_{3}$ crystals are dominated by the first order peaks. Two distinct lines of the $\mathrm{TO}_{1}$ phonons, the soft modes of $A$ and $E$ symmetries, are seen in the low-frequency range (See Fig. 1(a), their frequencies depend strongly on temperature and composition (Fig. 1(b)). Figure 2 shows the variation of the hard mode frequencies with Ba content $x$ at $\mathrm{T}=5 \mathrm{~K}$.

The two components of the $\mathrm{TO}_{1}$ soft phonon, the $E$ and $A_{1}$ modes have been observed at low temperatures for all $\mathrm{Ba}_{x} \mathrm{Sr}_{1-x} \mathrm{TiO}_{3}$ compositions studied. We found that for low $\mathrm{Ba}$ content $(x<0.2)$ the soft modes extrapolate to zero at $T_{c}$ and remain underdamped, indicating a displacive type of the ferroelectric phase transition. Heavy damping of the soft mode and the intensive low-frequency Raman scattering related to the order-disorder behavior are observed in $\mathrm{Ba}_{x} \mathrm{Sr}_{1-x} \mathrm{TiO}_{3}$ crystals with $x \geq 0.2$ in the temperature and composition range of the orthorhombic and tetragonal phases. There is no soft mode overdamping in the rhombohedral phase, which is completely ordered. No first-order Raman scattering in the cubic phase of $\mathrm{Ba}_{x} \mathrm{Sr}_{1-x} \mathrm{TiO}_{3}$ is observed for all compositions studied $(x \leq 0.5)$, in contrast to pure $\mathrm{BaTiO}_{3}$. Therefore, we conclude that the behavior characteristic for the order-disorder phase transitions becomes less noticeable with decreasing $\mathrm{Ba}$ content, and $\mathrm{Ba}_{x} \mathrm{Sr}_{1-x} \mathrm{TiO}_{3}$ with $x \lesssim 0.2$ can be considered as predominantly displacive type ferroelectrics with single ferroelectric phase and well-defined soft mode in the entire temperature range. Also, it can be seen on Fig. 1 that the $\mathrm{TO}_{1} A_{1}$ soft mode frequency decreases significantly and tends to merge with the $E$ mode when temperature approaches $T_{c}$ from below in $\mathrm{Ba}_{x} \mathrm{Sr}_{1-x} \mathrm{TiO}_{3}$ crystals with $x \leq 0.2$, but the $A_{1}$ mode shows much less softening in crystals with higher Ba content. This indicates that the polarization is discontinuous at $T_{c}$ in crystals with $x>0.2$, but the discontinuity apparently disappears for smaller $x$, i.e. the ferroelectric phase transition known to be of the first order for pure $\mathrm{BaTiO}_{3}$, is also of the first order in $\mathrm{Ba}_{x} \mathrm{Sr}_{1-x} \mathrm{TiO}_{3}$ with high $\mathrm{Ba}$ content, but turns into the second order for $x<0.2$. These single crystal results can be used as a reference point for the understanding of the lattice dynamical properties in $\mathrm{Ba}_{x} \mathrm{Sr}_{1-x} \mathrm{TiO}_{3}$ ceramics, thin films and nanostructures. 
This is an author-produced, peer-reviewed version of this article. The final, definitive version of this document can be found online at Journal of the

American Ceramic Society, published by Blackwell Publishing. Copyright restrictions may apply. doi: 10.1111/j.1551-2916.2008.02371.x

\section{RAMAN SPECTROSCOPY OF FERROELECTRIC THIN FILMS USING CONDUCTIVE OXIDE LAYERS}

In this section we review the results of Raman spectroscopy of $\mathrm{SrTiO}_{3}, \mathrm{Ba}_{x} \mathrm{Sr}_{1-x} \mathrm{TiO}_{3}$, and $\mathrm{BaTiO}_{3}$ films, obtained using the metal-oxide bilayer technique. ${ }^{45,46}$ The films studied were grown by pulsed laser deposition on $\mathrm{SrTiO}_{3}$ and $\mathrm{LaAlO}_{3}$ (LAO) substrates. A KrF excimer laser operating at the wavelength of $248 \mathrm{~nm}$ was used for the deposition. Between the substrate and $\mathrm{Ba}_{x} \mathrm{Sr}_{1-x} \mathrm{TiO}_{3}$ film a $0.3 \mu$ m-thick conducting layer of $\mathrm{SrRuO}_{3}$ was deposited to screen the Raman signal from the substrate. Visible light (mostly $514.5 \mathrm{~nm}$ line of Argon ion laser) was used for excitation. The thickness of all $\mathrm{BaTiO}_{3}$ and $\mathrm{Ba}_{x} \mathrm{Sr}_{1-x} \mathrm{TiO}_{3}$ films and most of $\mathrm{SrTiO}_{3}$ studied was $1 \mu \mathrm{m}$, but for some $\mathrm{SrTiO}_{3}$ films varied from 0.5 to $2.3 \mu \mathrm{m}$. These studies revealed essential differences in the lattice dynamical properties of $\mathrm{SrTiO}_{3}$, $\mathrm{Ba}_{x} \mathrm{Sr}_{1-x} \mathrm{TiO}_{3}$ thin films compared to single crystals.

\section{A. $\mathrm{SrTiO}_{3}$ films}

Raman spectra of $\mathrm{SrTiO}_{3}$ thin films obtained using the metal-oxide bilayer technique are very different from those of bulk $\mathrm{SrTiO}_{3}$ crystals. ${ }^{46}$ In Fig. 3, the spectra for three $\mathrm{SrTiO}_{3}$ films of different thicknesses measured at $\mathrm{T}=5 \mathrm{~K}$ are shown together with the spectrum of an $\mathrm{SrTiO}_{3}$ single crystal. By comparing with the hyper-Raman results of bulk single crystals, ${ }^{34}$, the strong peaks at 170 and $545 \mathrm{~cm}^{-1}$ were identified as due to the $\mathrm{TO}_{2}$ and $\mathrm{TO}_{4}$ phonons, respectively, and the weak peak at $264 \mathrm{~cm}^{-1}$ - to the silent $\mathrm{TO}_{3}$ mode. These peaks can be observed up to room temperature. In contrast, the Raman spectrum of the $\mathrm{SrTiO}_{3}$ single crystal is characterized by the second-order scattering features and the structural modes. (Weak $\mathrm{TO}_{2}$ and $\mathrm{TO}_{4}$ peaks can also be seen in the single crystal spectrum, likely due to the impurities in the sample). The appearance of the strong first-order TO phonon peaks in the thin film spectra indicates a lowering of the crystal symmetry in the films. (Spectra contain also peaks due to the $\mathrm{SrRuO}_{3}$ buffer layers at 98, 130, 230, 252, 361,393, and $412 \mathrm{~cm}^{-1}$, which are marked by stars. The same lines were observed in the spectra of bare $\mathrm{SrRuO}_{3}$ films and also reported in the literature. ${ }^{82}$ Similar peaks have also been observed in the spectra of $\mathrm{BaSrTiO}_{3}$ films grown on $\mathrm{SrRuO}_{3}$ buffer layers bilayer structures, described in the next subsection.) 
This is an author-produced, peer-reviewed version of this article. The final, definitive version of this document can be found online at Journal of the American Ceramic Society, published by Blackwell Publishing. Copyright restrictions may apply. doi: 10.1111/j.1551-2916.2008.02371.x

The metal-oxide bilayer method was also employed for studies of $\mathrm{SrTiO}_{3}$ films by farinfrared ellipsometry using synchrotron radiation. ${ }^{52}$ This technique combines ellipsometry with the high brightness of the synchrotron radiation, and provides a powerful capability to measure vibrational properties with high reliability and accuracy. The soft $\mathrm{TO}_{1}$ phonon modes were clearly observed along with other TO modes. The soft phonon frequency square, $\omega_{T O 1}^{2}$ is plotted as a function of temperature in Fig. 4, which also shows the inverse dielectric constant, $1 / \varepsilon_{0}$, as obtained from the low-frequency dielectric measurements. The results for a $\mathrm{SrTiO}_{3}$ film and a $\mathrm{SrTiO}_{3}$ single crystal are displayed for comparison. As shown by Fig. 4(a), the frequency of the soft-mode decreases as the temperature is lowered. However, in clear contrast to the bulk crystals where the frequency of the $\mathrm{TO}_{1}$ phonon mode saturates at $13 \mathrm{~cm}^{-1}$ at low temperature, ${ }^{83}$ in our $\mathrm{SrTiO}_{3}$ thin film the $\mathrm{TO}_{1}$ frequency remains fairly high and saturates at $62 \mathrm{~cm}^{-1}$. Since the frequencies of the hard modes (the phonons other than the soft mode) change only weakly with temperature and are completely the same as those in the bulk $\mathrm{SrTiO}_{3}$, a higher soft-mode frequency at low temperatures should lead to a lower the static dielectric constant $\varepsilon_{0}$, which is indeed observed as shown by Fig. 4(b). This observation demonstrates that the primary reason for low dielectric constant in $\mathrm{SrTiO}_{3}$ thin films is the soft mode hardening.

Studies of the response of the soft mode to external electric field proved to be very helpful for the understanding of the different field tuning behaviors of $\varepsilon_{0}$ in $\mathrm{SrTiO}_{3}$ thin films and crystals. In $\mathrm{SrTiO}_{3}$ single crystals, the dielectric nonlinearity vanishes above $\mathrm{T} \sim 80 \mathrm{~K}^{84}$ but in thin films, it remains non-zero even at room temperature. ${ }^{85}$ Fig. 5 shows Raman spectra of $\mathrm{SrTiO}_{3}$ thin films obtained at $10 \mathrm{~K}$ with and without application of external electric field. ${ }^{47}$ The phonon frequencies determined by Raman scattering are similar to the results of FTIR ellipsometry. Raman spectrum at zero field contains a strong peak at about $40 \mathrm{~cm}^{-1}$ originated from the $E_{u}$ component of the soft mode (labelled $E$ ). Application of the external electric field leads to appearance of the $A_{2 u}$ component of the soft mode (labelled $A$ ). The splitting between the $A$ and $E$ components od the soft mode at low temperatures and in the absence of electric field is induced by the tetragonal distortion (antiferrodistortive transition). The soft mode frequency in the film is higher at low temperature than that in bulk $\mathrm{SrTiO}_{3}$ crystals, in a quantitative agreement with the LST formalism.

Raman spectra of $\mathrm{SrTiO}_{3}$ films measured as a function of external electric field show that the soft mode frequency increases when an electric field is applied, and the electric field 
This is an author-produced, peer-reviewed version of this article. The final, definitive version of this document can be found online at Journal of the

American Ceramic Society, published by Blackwell Publishing. Copyright restrictions may apply. doi: 10.1111/j.1551-2916.2008.02371.x

induced soft-mode hardening is observed in the entire temperature range of the measurement, as shown on Fig. 6 Observed electric-field and temperature dependencies for $\varepsilon_{0}$ and $1 / \omega_{T O 1}^{2}$ are remarkably similar. ${ }^{47}$ Hence the mechanism of electric-field tunability of the dielectric constant in the $\mathrm{SrTiO}_{3}$ films is, as in the bulk, the field induced hardening of the soft mode. The difference in the dielectric nonlinearity in thin films and bulk crystals is due to the different soft-mode properties and their responses to the external electric field.

Petzelt et al. ${ }^{86-88}$ studied $\mathrm{SrTiO}_{3}$ ceramics, $\mathrm{SrTiO}_{3}$ and $\mathrm{Ba}_{x} \mathrm{Sr}_{1-x} \mathrm{TiO}_{3}$ films by Fourier transform infrared and Raman spectroscopy. They also observed important differences in the soft mode behavior of thin films compared to the bulk crystals, such as the soft mode hardening in thin films and presence of the symmetry-forbidden first order Raman peaks in the spectra of paraelectric phase. Similarly to $\mathrm{SrTiO}_{3}$ films, the lattice dynamical properties of $\mathrm{Ba}_{x} \mathrm{Sr}_{1-x} \mathrm{TiO}_{3}$ films were found to be essentially different from bulk $\mathrm{Ba}_{x} \mathrm{Sr}_{1-x} \mathrm{TiO}_{3}$, as discussed in the next subsection.

\section{B. $\mathrm{Ba}_{x} \mathrm{Sr}_{1-x} \mathrm{TiO}_{3}$ films}

There have been several Raman studies of $\mathrm{Ba}_{x} \mathrm{Sr}_{1-x} \mathrm{TiO}_{3}$ thin films, whose lattice dynamical properties are influenced by strain and defects in the films. Yuzyuk et al. ${ }^{41-44}$ reported several Raman studies of Ba-rich $(x \geq 0.55) \mathrm{Ba}_{x} \mathrm{Sr}_{1-x} \mathrm{TiO}_{3}$ films grown on $\mathrm{MgO}$ substrates, focusing mainly on the strain effects on the ferroelectric phase transitions in films. They observed the soft phonon modes of thin films and studied the influence of composition variation on the soft modes. Kim et $a l .{ }^{89}$ investigated the effect of crystallinity on the microwave dielectric losses in $\mathrm{Ba}_{x} \mathrm{Sr}_{1-x} \mathrm{TiO}_{3}$ films and found the correlation between the increased dielectric loss and the symmetry-breaking defects evidenced by Raman spectra.

We conducted a detailed Raman study of vibrational spectra of $\mathrm{Ba}_{x} \mathrm{Sr}_{1-x} \mathrm{TiO}_{3}$ films with Ba contents $x$ varied from 0.05 to 0.5 in comparison with single crystals of the same compositions. ${ }^{49,51}$ The films were grown by pulsed laser deposition on $\mathrm{SrTiO}_{3}$ substrates covered by $0.3 \mu \mathrm{m}$-thick conducting $\mathrm{SrRuO}_{3}$ layers. Temperature dependence of the phonon frequencies and intensities was investigated, with particular emphasis on the soft mode behavior. Significant differences in the soft mode behavior of the thin films and crystals are observed. We compare the vibrational properties of the $\mathrm{Ba}_{x} \mathrm{Sr}_{1-x} \mathrm{TiO}_{3}$ films with those of relaxor ferroelectrics, recently studied by inelastic neutron scattering. ${ }^{90,91}$ Remarkable 
This is an author-produced, peer-reviewed version of this article. The final, definitive version of this document can be found online at Journal of the

American Ceramic Society, published by Blackwell Publishing. Copyright restrictions may apply. doi: 10.1111/j.1551-2916.2008.02371.x

similarities are found between $\mathrm{Ba}_{x} \mathrm{Sr}_{1-x} \mathrm{TiO}_{3}$ thin films and relaxors, which are explained by the existence of polar nanoregions in the $\mathrm{Ba}_{x} \mathrm{Sr}_{1-x} \mathrm{TiO}_{3}$ thin films, analogously to those in relaxors.

All the $\mathrm{Ba}_{x} \mathrm{Sr}_{1-x} \mathrm{TiO}_{3}$ compositions studied are paraelectric at room temperature. ${ }^{54}$ All the zone-center optical phonon modes are forbidden in the first order Raman scattering in the cubic $m 3 m$ phase, but become Raman-active upon transition into a ferroelectric phase. Fig. 7 shows the Raman spectra for a film and a crystal with $x=0.2$ at 200 and $10 \mathrm{~K}$, above and below the ferroelectric phase transition $\left(120 \mathrm{~K}\right.$ in the bulk $\left.{ }^{54}\right)$. At low temperatures the spectra of both single crystal and film contain the lines of hard phonon modes $(178,276$, and $542 \mathrm{~cm}^{-1}$ in the film and $174,275,541 \mathrm{~cm}^{-1}$ in the crystal). These lines are attributed to the mixed $\mathrm{TO}_{2}+\mathrm{LO}_{1}, \mathrm{TO}_{3}+\mathrm{LO}_{2}$, and $\mathrm{TO}_{4}$ phonon modes, respectively. Similar lines were observed for $\mathrm{SrTiO}_{3}$ films, ${ }^{46}$ as discussed in the previous subsection. Above the ferroelectric phase transition the first order Raman lines disappear from the single crystal spectra, which contain only the broad features of the second order Raman scattering. However, in thin films the first order Raman lines are still seen even when the temperature is well above the phase transition, in violation of the symmetry selection rules. The same behavior was observed in films of all Ba concentrations studied.

Two lines of the $\mathrm{TO}_{1} A_{1}$ and $E$ soft modes were observed at low temperatures in the low-frequency spectral region of both crystals and films of all compositions studied, as shown in Fig. 7 for $\mathrm{Ba}_{0.2} \mathrm{Sr}_{0.8} \mathrm{TiO}_{3}$. They originate from the triple degenerated soft phonon of $F_{1 u}$ symmetry in the cubic phase. The $A_{1}$ mode appears in the ferroelectric phase only, which is used to determine the ferroelectric transition temperature. It has atomic displacements parallel to the $z$ axis, and its frequency is related to the spontaneous polarization. The lowest-frequency mode, $E$ soft mode has atomic displacements perpendicular to the polarization axis $z$, and the square of its frequency is inversely proportional to the dielectric constant in the $x y$ plane. ${ }^{26,27}$ It transforms continuously to the $F_{1 u}$ mode of the cubic phase when the temperature is increased to above the ferroelectric transition. The soft modes in $\mathrm{Ba}_{x} \mathrm{Sr}_{1-x} \mathrm{TiO}_{3}$ films appear at significantly higher frequencies than in single crystals. In crystals the soft mode frequencies decrease rapidly as the temperature approaches the ferroelectric phase transition from below. In films the soft modes show much less softening. ${ }^{49,51}$

Similarly to the $\mathrm{SrTiO}_{3}$ films discussed above, there are important differences in the phonon spectra of $\mathrm{Ba}_{x} \mathrm{Sr}_{1-x} \mathrm{TiO}_{3}$ films compared to the single crystals, such as the soft 
This is an author-produced, peer-reviewed version of this article. The final, definitive version of this document can be found online at Journal of the American Ceramic Society, published by Blackwell Publishing. Copyright restrictions may apply. doi: 10.1111/j.1551-2916.2008.02371.x

mode hardening and the presence of the first-order Raman peaks in the spectra of films at the temperatures above the ferroelectric phase transition temperature, indicating the breakdown of the inversion symmetry. The same behavior was observed in films of all $\mathrm{Ba}$ concentrations studied. Another difference in the soft mode behavior of $\mathrm{Ba}_{x} \mathrm{Sr}_{1-x} \mathrm{TiO}_{3}$ films and crystals appears when considering the $E$ soft mode damping. For larger Ba compositions $(x \geq 0.2)$, both in crystals and films the $E$ soft modes are not observable near the ferroelectric transition due to overdamping. The latter term means that the phonon linewidth is higher than the mode frequency itself, so there is no distinct peak at finite frequency in Raman spectra. The overdamping in $\mathrm{Ba}_{0.2} \mathrm{Sr}_{0.8} \mathrm{TiO}_{3}$ crystal and film is illustrated in Fig. 8, showing the temperature dependence of the linewidth (damping) for the $E$ soft mode shown in in comparison with the mode frequency. As one can see, the range of the soft mode overdamping is significantly broader in the film compared to the single crystal.

The soft mode mode is known to be heavily overdamped in pure bulk $\mathrm{BaTiO}_{3}$ at the temperatures around $T_{c}{ }^{25-27}$ due to the order-disorder character of the ferroelectric phase transition and the existence of so called central peak. ${ }^{66-68,71,73}$ Similar behavior is expectable to take place in $\mathrm{Ba}_{x} \mathrm{Sr}_{1-x} \mathrm{TiO}_{3}$, especially for Ba-rich compositions. Indeed, we have observed the soft mode overdamping in $\mathrm{Ba}_{x} \mathrm{Sr}_{1-x} \mathrm{TiO}_{3}$ crystals and films with $x \geq 0.2$, but there is a quantitative difference between the films and crystals. In Fig. 9, the range of overdamping is shown for both thin films (vertical error bars) and single crystals (shaded area) in comparison with the phase diagram for bulk $\mathrm{Ba}_{x} \mathrm{Sr}_{1-x} \mathrm{TiO}_{3} .{ }^{54}$ The ferroelectric phase transition temperatures shown here are determined by the disappearance of the $A_{1}$ soft mode. As can be seen on the Fig.9, the overdamping in the crystals occurs mainly in the orthorhombic and tetragonal phases. The $E$ soft mode is not overdamped in the rhombohedral phase, and there is no Raman-active modes above $T_{c}$ in the cubic phase. The range of overdamping is significantly broader in the films. This fact suggests that there are additional damping mechanisms in the films that are not present in the single crystals.

In order to explain the specific features of lattice vibrations in $\mathrm{Ba}_{x} \mathrm{Sr}_{1-x} \mathrm{TiO}_{3}$ films, making them essentially different from the bulk crystals, let us consider briefly the lattice dynamics behavior in relaxor ferroelectrics. Recently the lattice dynamics was studied by inelastic neutron scattering in relaxors, such as $\left.\mathrm{Pb}\left(\mathrm{Zn}_{1 / 3}\right) \mathrm{Nb}_{2 / 3}\right)_{1-x} \mathrm{Ti}_{x} \mathrm{O}_{3}(\mathrm{PZN}-x \mathrm{PT})^{92}$ and $\mathrm{Pb}\left(\mathrm{Mg}_{1 / 3} \mathrm{Nb}_{2 / 3}\right) \mathrm{O}_{3}(\mathrm{PMN}) .{ }^{90,91,93}$ The results of these studies, demonstrating the specific behavior of the soft phonon modes, supported the idea of polar nanoregions (PNR) in re- 
This is an author-produced, peer-reviewed version of this article. The final, definitive version of this document can be found online at Journal of the American Ceramic Society, published by Blackwell Publishing. Copyright restrictions may apply. doi: 10.1111/j.1551-2916.2008.02371.x

laxors. PNR are the nanometer-size regions of randomly oriented polarization, embedded in the non-polar background, giving rise to broad maxima in the temperature dependence of the dielectric constant with a characteristic frequency dependence. ${ }^{94,95}$ Burns and Dacol $^{95}$ demonstrated that a randomly oriented local polarization starts to develop at a certain temperature $T_{d}$ (the Burns temperature), several hundred degrees above the temperature of the dielectric constant maximum.

The neutron scattering results ${ }^{90,91}$ show that the soft mode in PMN is overdamped in a broad temperature range, but it is well resolved above the Burns temperature $T_{d}$ and below a temperature coincident with the field-induced ferroelectric transition temperature. The observed soft mode exhibits a normal ferroelectric behavior, i.e. the square of its frequency decreases linearly as the temperature approaches the phase transition. ${ }^{90,91}$ These behaviors are strikingly similar to the results on $\mathrm{Ba}_{x} \mathrm{Sr}_{1-x} \mathrm{TiO}_{3}$ films described in the present paper.

The soft mode overdamping, as well as the anomalous TA-phonon broadening in PMN ${ }^{91,93}$ was attributed to the presence of polar nanoregions, which couple strongly with the polar soft modes. ${ }^{91,95}$ We suggest that the similarities between the soft mode behaviors in bulk relaxor ferroelectrics and thin films of classical ferroelectric $\mathrm{Ba}_{x} \mathrm{Sr}_{1-x} \mathrm{TiO}_{3}$ indicate that the PNR also exist in $\mathrm{Ba}_{x} \mathrm{Sr}_{1-x} \mathrm{TiO}_{3}$ films in a broad temperature range. Strong coupling of the PNR with the polar soft phonons causes the damping in addition to the intrinsic damping mechanism in $\mathrm{Ba}_{x} \mathrm{Sr}_{1-x} \mathrm{TiO}_{3}$ due to the order-disorder behavior. Electric field associated with PNR causes the increase of the soft mode frequencies in the $\mathrm{Ba}_{x} \mathrm{Sr}_{1-x} \mathrm{TiO}_{3}$ films compared to single crystals, similarly to the soft mode hardening induced by external electric field in $\mathrm{SrTiO}_{3} \cdot{ }^{33,47}$ Also, the PNR locally break the inversion symmetry in $\mathrm{Ba}_{x} \mathrm{Sr}_{1-x} \mathrm{TiO}_{3}$ films, making the first order Raman scattering possible at temperatures above the ferroelectric phase transition.

The analogy in the lattice dynamics behavior of $\mathrm{Ba}_{x} \mathrm{Sr}_{1-x} \mathrm{TiO}_{3}$ films and relaxor ferroelectric PMN is illustrated in the Fig. 10. Both relaxors and $\mathrm{Ba}_{x} \mathrm{Sr}_{1-x} \mathrm{TiO}_{3}$ films are characterized by a broad maximum in the temperature dependence of the dielectric constant. For PMN, the deviation of $\varepsilon$ from the Curie-Weiss behavior occurs below the Burns temperature, ${ }^{96}$ which is much higher than the maximum in the $\varepsilon(T)$ dependence. Below $T_{d}$ correlations between PNR become important. ${ }^{96}$ Analogous behaviors are observed in $\mathrm{Ba}_{x} \mathrm{Sr}_{1-x} \mathrm{TiO}_{3}$ films as demonstrated in Fig. 10(a), the temperature dependence of $\varepsilon^{-1}$ for $\mathrm{Ba}_{0.2} \mathrm{Sr}_{0.8} \mathrm{TiO}_{3}$, which is very similar to the $\varepsilon^{-1}(T)$ dependence for PMN (Fig. 2 in the paper 
This is an author-produced, peer-reviewed version of this article. The final, definitive version of this document can be found online at Journal of the American Ceramic Society, published by Blackwell Publishing. Copyright restrictions may apply. doi: 10.1111/j.1551-2916.2008.02371.x

by Vieland et al. $\left.{ }^{96}\right)$. As in $\mathrm{PMN}, \varepsilon(T)$ in $\mathrm{Ba}_{x} \mathrm{Sr}_{1-x} \mathrm{TiO}_{3}$ films deviates from the Curie-Weiss law below a temperature $T_{d}$, which is much higher than the maximum of $\varepsilon(T)$.

Fig. 10(b) shows the temperature dependence of $\omega_{T O}^{2}$, the square of the $E$ or $F_{1 u}$ soft mode frequency. Comparing the figures 10 (a) and (b), one can see that above $T_{d}$ the dielectric constant is inversely proportional to $\omega_{T O}^{2}$ following the Lyddane-Sachs-Teller relation. The soft mode becomes overdamped below $T_{d}$, but is recovered at low temperatures, as shown on Fig. 10(c). Again, this behavior is very similar to that of PMN (Fig.4(a) from Ref. 9).

The existence of polar nanoregions in ferroelectric films explains also the observations of the first order Raman scattering in $\mathrm{SrTiO}_{3}$ films, ${ }^{46,47}$ and the earlier results obtained on $\mathrm{KTaO}_{3}$ crystals doped with $\mathrm{Li}$ and $\mathrm{Nb}$. Pure $\mathrm{KTaO}_{3}$, like $\mathrm{SrTiO}_{3}$ is incipient ferroelectric, which remains in the cubic paraelectric state down to low temperatures and has no Raman-active phonon modes. In doped $\mathrm{KTaO}_{3}$, the observations of the first order Raman scattering, ${ }^{97}$ the soft mode hardening, and the increased damping ${ }^{98}$ were attributed to the presence of PNR induced by off-center $\mathrm{Li}$ and $\mathrm{Nb}$ ions. In $\mathrm{Ba}_{x} \mathrm{Sr}_{1-x} \mathrm{TiO}_{3}$ films, recent investigations by time-resolved confocal scanning optical microscopy demonstrated the polarization reorientation of the polar nanoregions under electric field ${ }^{99}$ and a strong dispersion of microwave dielectric response due to the size-dependent relaxation frequencies of PNR. ${ }^{100}$ The similarities in the lattice dynamical properties between $\mathrm{Ba}_{x} \mathrm{Sr}_{1-x} \mathrm{TiO}_{3}$ films and relaxor ferroelectric PMN provide further evidences to the importance of polar nanoregions in determining the properties of ferroelectric thin films.

The characteristic feature of relaxor ferroelectrics is the frequency dispersion of temperature-dependent dielectric susceptibility in the hertz-gigahertz range. ${ }^{94}$ An important difference between relaxors and $\mathrm{Ba}_{x} \mathrm{Sr}_{1-x} \mathrm{TiO}_{3}$ thin films is the absence of such a dispersion in $\mathrm{Ba}_{x} \mathrm{Sr}_{1-x} \mathrm{TiO}_{3}$ films. This may be due to the different structure and size of PNR, and strain in thin films, which influence the polarization dynamics in PNR. In relaxors the formation of PNR is caused by the compositional heterogeneity resulting from the B site being occupied by ions of different valence (either $\mathrm{Mg}^{2+}$ or $\mathrm{Zn}^{2+}$, and $\mathrm{Nb}^{5+}$ ). ${ }^{94}$ In $\mathrm{Ba}_{x} \mathrm{Sr}_{1-x} \mathrm{TiO}_{3}$ films the origin of the PNR is more likely polar grain boundaries, oxygen vacancies and inhomogeneous strain. It should be noted that dielectric dispersion in $\mathrm{Ba}_{x} \mathrm{Sr}_{1-x} \mathrm{TiO}_{3}$ films has been observed in the terahertz range by Hubert et al. in resemblance with those of relaxors. $^{100}$

Several possible causes for the formation of the polar nanoregions in ferroelectric thin films 
This is an author-produced, peer-reviewed version of this article. The final, definitive version of this document can be found online at Journal of the American Ceramic Society, published by Blackwell Publishing. Copyright restrictions may apply. doi: 10.1111/j.1551-2916.2008.02371.x

were suggested. In polycrystalline $\mathrm{SrTiO}_{3}$ films Ostapchuk et al. ${ }^{88}$ observed such defects as porosity and cracks along the grain boundaries and suggested these defects to cause the appearance of of symmetry forbidden Raman peaks and soft mode hardening. However, these defects were not found in epitaxial films studied by Sirenko et al. ${ }^{46,47,52}$, as demonstrated by Wu et $a .^{101}$ Another cause for the appearance of polar nanoregions proposed by Petzelt et $a l{ }^{86}$ for $\mathrm{SrTiO}_{3}$ ceramics is the presence of dipoles localized at the grain boundaries, which can explain the observed soft mode hardening and presence of the symmetry-forbidden first order Raman peaks in the spectra of epitaxial $\mathrm{SrTiO}_{3}$ films. Other factors that can cause thin film properties to be different from bulk, include nonstoichiometry, inhomogeneous strain distribution, impurities and oxygen vacancies, which are among the most common defects in oxide thin films. ${ }^{102}$ Uwe et al. have shown that introducing oxygen vacancies into a nominally pure $\mathrm{SrTiO}_{3}$ crystal leads to appearance of local regions of ferroelectric polarization. ${ }^{103}$ Infrared reflectance studies of highly reduced $\mathrm{SrTiO}_{3}$ showed the presence of oxygen vacancies to cause hardening of the TO phonon. ${ }^{104}$ It is important to know, which of these factors plays a more significant role in determining the thin films behavior.

In order to investigate the effect of oxygen vacancies on the lattice dynamics of $\mathrm{SrTiO}_{3}$, we have studied oxygen reduced $\mathrm{SrTiO}_{3}$ single crystals by Raman spectroscopy. ${ }^{105}$ Comparing the Raman spectra of reduced $\mathrm{SrTiO}_{3}$ crystals and $\mathrm{SrTiO}_{3}$ films, we found that both in films and reduced $\mathrm{SrTiO}_{3}$ there are first order peaks of $\mathrm{SrTiO}_{3}$ phonons. This indicates that there are local regions where the inversion symmetry is broken, and $\mathrm{SrTiO}_{3}$ phonons become Raman active. In reduced $\mathrm{SrTiO}_{3}$, the oxygen vacancies, being polar defects, are responsible for the appearance of the polar regions. However, in reduced $\mathrm{SrTiO}_{3}$ the first order Raman peaks appear at rather high vacancy concentrations, where $\mathrm{SrTiO}_{3}$ becomes conductive. The appearance of the first-order phonon lines is accompanied by the peaks of local vibrational modes associated with oxygen vacancies. ${ }^{105}$ The latter modes have not been observed in $\mathrm{SrTiO}_{3}$ films, which are still insulating. ${ }^{85}$ Therefore, we conclude that although oxygen vacancies do cause the formation of polar nanoregions, in thin films the vacancy concentration is not high enough to cause such intensive first order Raman scattering. There must be another or additional cause for polar regions in $\mathrm{SrTiO}_{3}$ films. Polar impurities may lead to similar effects, but very high doping levels are needed (tenths of \%) to induce a significant first order Raman scattering, ${ }^{106,107}$, which is unlikely in nominally undoped films we studied. Sr/Ti non-stoichiometry could be another possible factor, but it is difficult to 
This is an author-produced, peer-reviewed version of this article. The final, definitive version of this document can be found online at Journal of the

American Ceramic Society, published by Blackwell Publishing. Copyright restrictions may apply. doi: 10.1111/j.1551-2916.2008.02371.x

prove the existence of a small amount of non-stoichiometry in thin films.

Strain in thin epitaxial films can be an important factor strongly affecting their properties $^{108}$ Transmission electron microscopy (TEM) studies were performed on $\mathrm{SrTiO}_{3} / \mathrm{SrRuO}_{3}$ epitaxial bilayers grown on $\mathrm{LaAlO}_{3}$ substrates, ${ }^{101}$ which Raman spectra are similar to those of $\mathrm{SrTiO}_{3} / \mathrm{SrRuO}_{3}$ films on $\mathrm{SrTiO}_{3}$. TEM results demonstrate that the lattice mismatch between the substrate and the $\mathrm{SrRuO}_{3}$ layer is mostly relaxed by dislocations in the vicinity of the $\mathrm{SrRuO}_{3} /$ substrate interface, although some defects propagate through the $\mathrm{SrRuO}_{3}$ layer and reach the $\mathrm{SrRuO}_{3} / \mathrm{SrTiO}_{3}$ interface, resulting in defects in the $\mathrm{SrTiO}_{3}$ layers. ${ }^{101}$ The lattice mismatch between $\mathrm{SrRuO}_{3}$ and $\mathrm{SrTiO}_{3}(0.64 \%)$ is small compared to that between $\mathrm{SrRuO}_{3}$ and $\mathrm{LaAlO}_{3}(3.6 \%)$, therefore one can expect a significantly smaller dislocation density in $\mathrm{SrTiO}_{3} / \mathrm{SrRuO}_{3}$ films on $\mathrm{SrTiO}_{3}$ substrates compared to the films on $\mathrm{LaAlO}_{3}$. However, Raman spectra are similar for the films grown on $\mathrm{SrTiO}_{3}$ and $\mathrm{LaAlO}_{3}$ substrates, both showing the forbidden first order Raman peaks. Therefore, we believe that dislocations are unlikely to cause the observed features in Raman spectra.

The small misfit between the $\mathrm{SrRuO}_{3}$ layer and the $\mathrm{SrTiO}_{3}$ film was shown to be mainly not relaxed and accommodated by elastic strain in the $\mathrm{SrTiO}_{3}$ films. ${ }^{101} \mathrm{~A}$ uniform biaxial strain does not, however, break the inversion symmetry and cannot cause the observed Raman peaks in the films, unless the strain exceeds the critical value for inducing ferroelectricity, ${ }^{109}$ which is not the case in our films. Although we cannot rule out the influence of the inhomogeneous strain distribution in the films, we believe that the likely cause for the appearance of polar nanoregions is the dipoles localized at the grain boundaries in films. The polar grain boundaries were proposed by Petzelt et al. ${ }^{86}$ to explain the observed the soft mode hardening and appearance of the first order Raman peaks of considerable intensity in $\mathrm{SrTiO}_{3}$ ceramics. The ceramics studied were nominally pure, highly stoichiometric and not subject to strain, yet the first order Raman peaks were observed. Ostapchuk et $a l .{ }^{88}$ studied polycrystalline $\mathrm{SrTiO}_{3}$ films and also observed the activation of the forbidden modes in the Raman spectra in the whole temperature range studied ( $80-300 \mathrm{~K})$, which was explained by the dipoles associated with such defects as porosity and cracks along the grain boundaries. Although these defects were not found in epitaxial films studied here, the dipoles frozen at the grain boundaries can still exist and induce the polar phase in the bulk of grains, leading to the symmetry breakdown and appearance of the forbidden modes in the Raman spectra, as well as the soft mode hardening and increased damping. 
This is an author-produced, peer-reviewed version of this article. The final, definitive version of this document can be found online at Journal of the American Ceramic Society, published by Blackwell Publishing. Copyright restrictions may apply. doi: 10.1111/j.1551-2916.2008.02371.x

\section{Strain effect on the phase transitions in $\mathrm{BaTiO}_{3}$ films}

As discussed in the Section II, bulk $\mathrm{BaTiO}_{3}$ undergoes three phase transitions, in which the crystal structure changes from cubic to tetragonal at $403 \mathrm{~K}$, then to orthorhombic at $278 \mathrm{~K}$, and to rhombohedral at $183 \mathrm{~K}$. The cubic phase is paraelectric and the other phases are ferroelectric. In thin films, strain due to lattice and/or thermal expansion mismatch between a film and a substrate may result in dramatic changes in phase transition behavior. ${ }^{108}$ Raman spectroscopy is a powerful tool to study phase transitions as the structural changes alter the vibrational spectra. We applied this technique to study phase transitions in epitaxial $\mathrm{BaTiO}_{3}$ thin $(1 \mu \mathrm{m})$ films grown by pulsed laser deposition on $\mathrm{SrTiO}_{3}$ and $\mathrm{LaAlO}_{3}$ substrates covered by $0.3 \mu \mathrm{m}$-thick conducting $\mathrm{SrRuO}_{3}$ layers, ${ }^{50}$ focusing on the temperature range 5-325 K, where the transitions from tetragonal to orthorhombic and rhombohedral phase take place in bulk $\mathrm{BaTiO}_{3}$.

Fig. 11 shows Raman spectra of a $\mathrm{BaTiO}_{3}$ single crystal and a film grown on a $\mathrm{SrTiO}_{3}$ substrate as a function of temperature. The spectra of the single crystal show clear changes attributed to the phase transitions, most noticeable in the frequency range $150-300 \mathrm{~cm}^{-1}$. At low temperatures, in the rhombohedral phase, the most intensive lines observed in the polarized Raman spectrum are at 173, 187, 242, 485, 522, and $714 \mathrm{~cm}^{-1}$, and are attributed to $\mathrm{TO}_{1}, \mathrm{LO}_{1}, \mathrm{TO}_{2}, \mathrm{LO}_{3}, \mathrm{TO}_{4}$, and $\mathrm{LO}_{4}$ modes of $A_{1}$ symmetry, respectively. ${ }^{29}$ The peak at 310 $\mathrm{cm}^{-1}$ is due to mixed $\mathrm{LO}_{2}-\mathrm{TO}_{3}$ phonon of $E$ symmetry. ${ }^{29}$ When the temperature is increased up to $\sim 185 \mathrm{~K}$, sudden jumps to a higher frequency, as large as $35 \mathrm{~cm}^{-1}$, took place for the $A_{1}\left(\mathrm{TO}_{2}\right)$ phonon line. A less dramatic, but still well pronounced jump $\left(\sim 10 \mathrm{~cm}^{-1}\right)$ occurred for the $A_{1}\left(\mathrm{TO}_{4}\right)$ line. These changes are indicative of the rhombohedral-orthorhombic phase transition. When the crystal was heated further to $\sim 280 \mathrm{~K}$, another sudden jumps of the $A_{1} \mathrm{TO}_{2}$ and $\mathrm{TO}_{4}$ phonon frequencies (25 and $4 \mathrm{~cm}^{-1}$, respectively) and changes in the Raman spectra occurred, which indicates the orthorhombic-tetragonal phase transition. The observed frequency shifts are well detectable in Raman spectra despite large linewidths $\mathrm{TO}_{2}$ and $\mathrm{TO}_{4}$ phonons, and are in agreement with the reported results for $\mathrm{BaTiO}_{3}$ crystals. ${ }^{24,30,31}$ The temperature dependence of the Raman spectra of the $\mathrm{BaTiO}_{3}$ film, shown in Fig. 11(b), is markedly different from that of the single crystal. It does not show sharp changes in the temperature range of 5-300 K. When temperature increases, the phonon lines broaden, but their frequencies are either almost constant $\left(\mathrm{TO}_{2}\right)$ or change only very slightly and gradually 
This is an author-produced, peer-reviewed version of this article. The final, definitive version of this document can be found online at Journal of the American Ceramic Society, published by Blackwell Publishing. Copyright restrictions may apply. doi: 10.1111/j.1551-2916.2008.02371.x

$\left(\mathrm{TO}_{4}\right)$. The two intensive sharp lines observed at 173 and $187 \mathrm{~cm}^{-1}$ at low temperatures (rhombohedral phase) in the $\mathrm{BaTiO}_{3}$ crystal are broader and their relative intensity is much smaller in the films at all temperatures measured. In fact, the positions, relative intensities and lineshapes of phonon modes in the spectra of the films are similar to those of the orthorhombic phase of the single crystal.

The temperature dependence of the frequencies of the $A_{1}\left(\mathrm{TO}_{2}\right)$ and $A_{1}\left(\mathrm{TO}_{4}\right)$ phonon modes for both the crystal and film shown on Fig. 12 clearly demonstrates the difference between the crystal and film behavior. Large jumps in the $A_{1}\left(\mathrm{TO}_{2}\right)$ phonon frequency are clearly seen in the crystal, but it is constant in the film. The $A_{1}\left(\mathrm{TO}_{4}\right)$ phonon frequency shows smaller jumps in the crystal, whereas no such jumps are visible in the film. This behavior indicates that the $\mathrm{BaTiO}_{3}$ film does not undergo any phase transition in the temperature range 5-325 K. The same behavior was observed for films grown both on $\mathrm{SrTiO}_{3}$ and $\mathrm{LaAlO}_{3}$ substrates. The tetragonal-orthorhombic and orthorhombic-rhombohedral phase transitions of the bulk $\mathrm{BaTiO}_{3}$ are completely absent in the thin films.

The observed behavior was attributed to the presence of a thermal-mismatch-induced tensile strain in the $\mathrm{BaTiO}_{3}$ films. For a quantitative determination of film strain the $\mathrm{BaTiO}_{3} / \mathrm{SrRuO}_{3}$ films studied were charachterized by the x-ray diffraction and transmission electron microscopy. X-ray data show that both the $\mathrm{BaTiO}_{3}$ and $\mathrm{SrRuO}_{3}$ layers grow epitaxially with $c$ axis normal to the substrate. The films are in-plane aligned with the substrate lattice with [100] $\mathrm{BaTiO}_{3}\left\|[100] \mathrm{SrRuO}_{3}\right\|[100] \mathrm{LaAlO}_{3} \cdot{ }^{50}$

From x-ray data, the lattice parameters of $\mathrm{SrRuO}_{3}$ buffer layers are determined to be $a=3.93 \AA$ for the pseudocubic lattice, equal to the bulk values, i.e. the $\mathrm{SrRuO}_{3}$ layers (both on $\mathrm{SrTiO}_{3}$ and $\mathrm{LaAlO}_{3}$ ) are completely relaxed. For $\mathrm{BaTiO}_{3}$ films the measured lattice constants are $a=b=4.01 \pm 0.01 \AA$ (in-plane), and $c=4.004 \pm 0.001 \AA$. Similar x-ray diffraction data were obtained for films grown on $\mathrm{SrTiO}_{3}$ substrates, yielding the same epitaxial orientation, the same $\mathrm{SrRuO}_{3}$ and $\mathrm{BaTiO}_{3}$ lattice constants. Transmission electron microscopy studies showed that the films consist of columnar grains with in-plane grain size of $\sim 100 \mathrm{~nm}$. Electron diffraction results indicated the $\mathrm{BaTiO}_{3}$ grains are oriented in same direction and confirmed the epitaxial relationship between the substrate, $\mathrm{SrRuO}_{3}$ and $\mathrm{BaTiO}_{3}$ layers described above. From the the lattice constants of the unstrained $\mathrm{BaTiO}_{3}$, the strain in the $\mathrm{BaTiO}_{3}$ thin films at room temperature was calculated to be a tensile strain of $0.58 \%$. Although the lattice constants of both $\mathrm{LaAlO}_{3}$ and $\mathrm{SrTiO}_{3}$ substrates and of $\mathrm{SrRuO}_{3}$ 
This is an author-produced, peer-reviewed version of this article. The final, definitive version of this document can be found online at Journal of the

American Ceramic Society, published by Blackwell Publishing. Copyright restrictions may apply. doi: 10.1111/j.1551-2916.2008.02371.x

buffer layer are smaller than that of $\mathrm{BaTiO}_{3}$, the misfit strain in the $\mathrm{BaTiO}_{3}$ film is fully relaxed at the growth temperatures because the film thickness $(1 \mu \mathrm{m})$ far exceeds the critical thickness for strain relaxation. Although the strain state in such a structure containing thick $\mathrm{BaTiO}_{3}$ and $\mathrm{SrRuO}_{3}$ layers is difficult to predict, the tensile strain in $\mathrm{BaTiO}_{3}$ films can arise from the thermal expansion mismatch. The thermal expansion coefficients of $\mathrm{LaAlO}_{3}{ }^{110}$ and $\mathrm{SrRuO}_{3}{ }^{111}$ are smaller than that of $\mathrm{BaTiO}_{3} \cdot{ }^{110}$ Consequently, when a $\mathrm{BaTiO}_{3}$ film is cooled to room temperature after the deposition, it contracts more than underlaying layers and a tensile stress is imposed on it. It is difficult to calculate the strain between 5-300 K because the thermal expansion coefficients are temperature dependent. However, the tensile strain is expected to increase when the temperature is lowered from 300 to 5 K. (Estimation assuming the room temperature strain of $0.58 \%$, as determined experimentally, and the temperature independent average thermal mismatch between $\mathrm{LaAlO}_{3}, \mathrm{SrRuO}_{3}$ and $\mathrm{BaTiO}_{3}$ results in $0.78 \%$ tensile strain at $5 \mathrm{~K}$.)

Comparison of the experimentally determined magnitude of the film strain $\mathrm{BaTiO}_{3}$ with the calculated temperature-strain phase diagram for $\mathrm{BaTiO}_{3}$ films under biaxial substrate constraint ${ }^{112}$ shows that a tensile strain of $0.58 \%$ at room temperature corresponds to the orthorhombic phase with in-plane polarization. This is confirmed by the Raman spectroscopy results (Figs. 11 and 12), as the form of the spectra, the phonon line positions and relative intensities in the $\mathrm{BaTiO}_{3}$ film are close to those of the orthorhombic phase of $\mathrm{BaTiO}_{3}$ single crystal. Because the tensile strain in the film increases when the temperature decreases from room temperature to $5 \mathrm{~K}$, the film remains in the orthorhombic phase without undergoing any phase transitions.

\section{UV RAMAN SPECTROSCOPY OF NANOSCALE FERROELECTRIC SU- PERLATTICES}

In the previous section we discussed some results of Raman studies of $\mathrm{SrTiO}_{3}$ and $\mathrm{Ba}_{x} \mathrm{Sr}_{1-x} \mathrm{TiO}_{3}$ films obtained using the metal-oxide bilayer technique. These studies revealed essential differences in the lattice dynamical properties of thin films compared to single crystals of the same materials. These results, along with the studies by other groups ${ }^{41-44,87-89}$ contributed significantly to a fundamental understanding of lattice dynamical properties in ferroelectric thin films. However, all these results were obtained on films or superlat- 
This is an author-produced, peer-reviewed version of this article. The final, definitive version of this document can be found online at Journal of the

American Ceramic Society, published by Blackwell Publishing. Copyright restrictions may apply. doi: 10.1111/j.1551-2916.2008.02371.x

tices ${ }^{113,114}$ thicker than $150 \mathrm{~nm}$.

Study of lattice dynamics in ferroelectric films and heterostructures thinner than $\sim 100 \mathrm{~nm}$ has been a challenging task. Conventional visible Raman spectroscopy works poorly for thin films of ferroelectrics and other wide-bandgap materials because the visible photon energy is much smaller than the bandgap (see Fig. 13(a) with $\mathrm{SrTiO}_{3}$ as an example). Consequently, the absorption of light is extremely weak and the penetration depth is large, allowing light to travel through thin film into the much thicker substrate, which generates overwhelming signals in the Raman spectra. The above described Raman studies of ferroelectric thin films had to employ an opaque layer of a conductive oxide, or study films grown on Ramaninactive substrates, such as $\mathrm{MgO}$. We have recently realized another approach using an UV excitation for Raman studies of nanoscale ferroelectrics. ${ }^{53}$ For UV excitation, the photon energy is above the bandgap of ferroelectric oxides such as $\mathrm{SrTiO}_{3} \mathrm{BaTiO}_{3}$, which leads to a much stronger absorption and a greatly reduced penetration depth (Fig. 13(a)). Thus the substrate contribution is suppressed, and phonons of nanoscale films and heterostructures can be observed. UV excitation near the bandgap can also lead to a resonance enhancement of Raman signals.

The advantage of UV over visible excitation is clearly demonstrated by Fig. 13(b) showing Raman spectra of a ferroelectric $\left[\left(\mathrm{BaTiO}_{3}\right)_{5} /\left(\mathrm{SrTiO}_{3}\right)_{4}\right] \times 25$ superlattice having total thickness of $\sim 90 \mathrm{~nm}$ measured with visible $(514.5 \mathrm{~nm})$ and UV (351.1 nm) excitations are shown. $\left(\mathrm{BaTiO}_{3} / \mathrm{SrTiO}_{3}\right.$ superlattices are denoted by $\left[\left(\mathrm{BaTiO}_{3}\right)_{n} /\left(\mathrm{SrTiO}_{3}\right)_{m}\right] \times$ number of periods, where $n$ and $m$ refer to the thickness, in unit cells, of the $\mathrm{BaTiO}_{3}$ and $\mathrm{SrTiO}_{3}$ layers, respectively.) The substrate features dominate the $514.5 \mathrm{~nm}$ spectrum while they are greatly reduced in the UV spectrum, in which peaks of superlattice phonons are clearly seen.

So far, UV Raman spectroscopy has not been widely used for ferroelectric films because of technical difficulties such as lower throughput efficiency, insufficient dispersion, and higher stray light level of UV Raman spectrometers compared to those operating in the visible range. Only a room temperature measurement of $\mathrm{SrTiO}_{3}$ films using $325 \mathrm{~nm}$ excitation has been reported ${ }^{115}$. Recent progress in UV Raman instrumentation has made measurement of ferroelectric ultrathin films possible. In our experiment, UV-optimized triple spectrometers (Jobin Yvon T64000) with a multichannel coupled charge-device detector were used. The system employs a triple monochromator to provide high resolution and effective stray light 
This is an author-produced, peer-reviewed version of this article. The final, definitive version of this document can be found online at Journal of the American Ceramic Society, published by Blackwell Publishing. Copyright restrictions may apply. doi: 10.1111/j.1551-2916.2008.02371.x

reduction. Powerful laser sources and optimized optical paths were used to improve the throughput. The $351.1 \mathrm{~nm}$ line of an $\mathrm{Ar}^{+}$laser and the $325 \mathrm{~nm}$ line of a He-Cd laser were used for excitation. Spectra were recorded in a backscattering geometry in the temperature range from 7 to $700 \mathrm{~K}$ using either a closed cycle cryostat (for low temperatures) or an evacuated heater stage (for above $300 \mathrm{~K}$ ). With these setups, we have measured Raman scattering in $\mathrm{BaTiO}_{3} / \mathrm{SrTiO}_{3}$ superlattices as thin as $24 \mathrm{~nm}$, and in singla-layer $\mathrm{BaTiO}_{3}$ films as thin as $2.4 \mathrm{~nm}$, i.e. 6 unit cells (See Fig. 13(c)).

We have demonstrated the UV Raman spectroscopy to be an effective technique to determine the phase transition temperature $T_{c}$ in ferroelectric ultrathin films and superlattices, which is a critical but challenging step for understanding ferroelectricity in nanoscale systems. The $T_{c}$ determination from Raman data is based on the fact that perovskite-type crystals have no first-order Raman active modes in paraelectric phase. We applied this method to study phase transitions in $\mathrm{BaTiO}_{3} / \mathrm{SrTiO}_{3}$ superlattices, as described below.

The $\left[\left(\mathrm{BaTiO}_{3}\right)_{n} /\left(\mathrm{SrTiO}_{3}\right)_{m}\right] \times N$ superlattice samples were grown by reactive molecularbeam epitaxy on $\mathrm{TiO}_{2}$-terminated (001) $\mathrm{SrTiO}_{3}$ substrates. The $n$ and $m$ values are controlled using reflection high-energy electron diffraction (RHEED) oscillations during growth, and confirmed by x-ray diffraction (XRD) and in some samples by high resolution transmission electron microscopy (HRTEM) ${ }^{116}$. XRD showed excellent epitaxy and crystallinity in the superlattice samples, with all superlattice peaks present in the $\theta-2 \theta$ scan. HRTEM images showed atomically sharp $\mathrm{BaTiO}_{3} / \mathrm{SrTiO}_{3}$ interfaces and acurate periodicity. ${ }^{116} \mathrm{Ac}-$ cording to XRD, all samples studied were commensurate to the $\operatorname{SrTiO}_{3}$ substrate $(a=0.3905$ $\mathrm{nm}$ ), meaning that the $\mathrm{SrTiO}_{3}$ layers are strain free and the $\mathrm{BaTiO}_{3}$ layers are under 2.2\% compressive biaxial strain ( $a=0.3992 \mathrm{~nm}$ in bulk $\mathrm{BaTiO}_{3}$ at room temperature), except for a $200 \mathrm{~nm}$-thick superlattice $\left[\left(\mathrm{BaTiO}_{3}\right)_{8} /\left(\mathrm{SrTiO}_{3}\right)_{4}\right] \times 40$, in which the strain is partially relaxed ( $a=0.3946 \mathrm{~nm}$ in-plane). In this case, the $\mathrm{SrTiO}_{3}$ layers are under biaxial tensile strain and the $\mathrm{BaTiO}_{3}$ layers are under biaxial compressive strain.

Fig. 14 shows the comparison of room temperature spectra of two superlattices, $\left(\mathrm{BaTiO}_{3}\right)_{8} /\left(\mathrm{SrTiO}_{3}\right)_{4} \times 10$ and $\left.\left(\mathrm{BaTiO}_{3}\right)_{5} /\left(\mathrm{SrTiO}_{3}\right)_{4}\right) \times 25,\left(T_{c}=640\right.$ and $530 \mathrm{~K}$, respectively) with the spectra of $\mathrm{BaTiO}_{3}$ single crystals in the three different ferroelectric phases (tetragonal, orthorhombic, and rhombohedral) and the $\mathrm{SrTiO}_{3}$ substrate spectra at 295 and $5 \mathrm{~K}$. The top two spectra are typical of the UV Raman spectra of $\mathrm{BaTiO}_{3} / \mathrm{SrTiO}_{3}$ superlattices below $T_{c}$, exhibiting strong first-order (single-phonon) peaks as labeled in the figure. 
This is an author-produced, peer-reviewed version of this article. The final, definitive version of this document can be found online at Journal of the American Ceramic Society, published by Blackwell Publishing. Copyright restrictions may apply. doi: 10.1111/j.1551-2916.2008.02371.x

Weak second-order (two-phonon) features from the $\mathrm{SrTiO}_{3}$ substrate can be seen between 600 and $700 \mathrm{~cm}^{-1}$ and as a background in the range $200-500 \mathrm{~cm}^{-1}$. The phonon mode assignment is made by comparison with the spectra of $\mathrm{SrTiO}_{3}$ and $\mathrm{BaTiO}_{3}$ single crystals and with the help of first-principles calculations.

The lines at about $290 \mathrm{~cm}^{-1}$ have similar positions and shapes to the $\mathrm{TO}_{2}$ modes of $A_{1}$ symmetry of the tetragonal-phase $\mathrm{BaTiO}_{3}{ }^{26,50}$, thus they are assigned to the $\mathrm{BaTiO}_{3}$ layers. We conclude that $\mathrm{BaTiO}_{3}$ layers in superlattices are tetragonal. This is supported by the absence of the sharp peak at $310 \mathrm{~cm}^{-1}$ characteristic of the orthorhombic and rhombohedral phases of $\mathrm{BaTiO}_{3}$, but not pronounced in the tetragonal phase.

The frequencies of several phonon branches in $\mathrm{SrTiO}_{3}$ and $\mathrm{BaTiO}_{3}$ are close to each other, and the phonons are not expected to be strongly localized within the thin $\mathrm{SrTiO}_{3}$ and $\mathrm{BaTiO}_{3}$ layers. According to first-principle calculations, these vibrations extend through the whole superlattice. This is the case for the $\mathrm{LO}_{3}$ and the $\mathrm{TO}_{4}$ modes, observed at about 478 and $530 \mathrm{~cm}^{-1}$, respectively.

The line at about $180 \mathrm{~cm}^{-1}$ is attributed to the $\mathrm{SrTiO}_{3}$-like $\mathrm{TO}_{2}$ phonons. Its position corresponds closely to the $\mathrm{TO}_{2}$ line in the electric-field-induced Raman spectrum of $\mathrm{SrTiO}_{3}$ crystals ${ }^{33}$ and the weak feature in the $5 \mathrm{~K}$ spectrum of the $\mathrm{SrTiO}_{3}$ single crystal. (The first-order Raman lines are visible in these cases because the electric field and defects break the inversion symmetry in the $\mathrm{SrTiO}_{3}$ crystals.) The $180 \mathrm{~cm}^{-1}$ line is not from the $\mathrm{SrTiO}_{3}$ substrate because the first-order Raman lines are symmetry-forbidden in bulk $\mathrm{SrTiO}_{3} \cdot{ }^{32} \mathrm{In}$ fact, even the much stronger second-order features of the substrate at 230 and $610 \mathrm{~cm}^{-1}$ are barely seen in the UV Raman spectra. Although $\mathrm{BaTiO}_{3}$ also has a feature due to the $\mathrm{TO}_{1}$ mode of $A_{1}$ symmetry at about the same position $\left(177 \mathrm{~cm}^{-1}\right)$, at room temperature (in the tetragonal phase) that feature is about 15-20 times weaker compared to the $\mathrm{TO}_{2}$ and $\mathrm{TO}_{4}$ lines, and it has a characteristic interference dip due to the coupling of the $\mathrm{TO}_{1}$ and $\mathrm{TO}_{2}$ modes of $A_{1}$ symmetry. ${ }^{26}$ The $180 \mathrm{~cm}^{-1}$ line observed in the spectra of the superlattices is of the same order of magnitude in intensity as the $\mathrm{TO}_{2}$ and $\mathrm{TO}_{4}$ lines of $\mathrm{BaTiO}_{3}$ and does not have the dip characteristic of $\mathrm{BaTiO}_{3}$. Therefore, although we cannot absolutely rule out the contribution of the $\mathrm{BaTiO}_{3}$ layers, we believe this line should be attributed to the phonons of the $\mathrm{SrTiO}_{3}$ layers in the superlattices. The observation of the first-order Raman scattering by $\mathrm{SrTiO}_{3}$ phonons indicates that the inversion symmetry is broken, and the $\mathrm{SrTiO}_{3}$ layers in the superlattices are polar. 
This is an author-produced, peer-reviewed version of this article. The final, definitive version of this document can be found online at Journal of the

American Ceramic Society, published by Blackwell Publishing. Copyright restrictions may apply. doi: 10.1111/j.1551-2916.2008.02371.x

A doublet of folded longitudinal acoustic (LA) phonon due to the superlattice periodicity $^{117}$ is also observed. The two triangles indicate the predicted first doublet frequencies by an elastic continuum model ${ }^{118}$. The observation of the LA phonon folding suggests that these superlattices possess the requisite structural quality for acoustic Bragg mirrors and cavities used for coherent phonon generation ${ }^{119,120}$.

Fig. 15 shows the temperature evolution of Raman spectra for two $\mathrm{BaTiO}_{3} / \mathrm{SrTiO}_{3}$ superlattices. From the shapes and positions of the $\mathrm{BaTiO}_{3}$ lines it follows that the $\mathrm{BaTiO}_{3}$ layers are in ferroelectric tetragonal phase at all temperatures below the $T_{c}$. The intensities of the first-order superlattice phonons decrease as the temperature increases and disappear at $T_{c}$. Above $T_{c}$, the spectra contain only the second-order features as expected from the symmetry selection rules. When the $\mathrm{BaTiO}_{3}$ layers are paraelectric, the induced polarization in the $\mathrm{SrTiO}_{3}$ layers also disappears.

By plotting the first-order Raman intensity as a function of temperature, we can determine $T_{c}$ accurately as the temperature where the intensity becomes zero. For this purpose, the $\mathrm{TO}_{2}$ and $\mathrm{TO}_{4}$ phonon lines are the most suitable because they do not overlap with the second-order features. The results, with the phonon intensities normalized by the Bose factor $n+1=(1-\exp (-\hbar \omega / k T))^{-1}$ and by the intensities at $7 \mathrm{~K}$, are presented for four superlattices: $\left[\left(\mathrm{BaTiO}_{3}\right)_{2} /\left(\mathrm{SrTiO}_{3}\right)_{4}\right] \times 40$ and $\left.\left[\left(\mathrm{BaTiO}_{3}\right)_{5} /\left(\mathrm{SrTiO}_{3}\right)_{4}\right)\right] \times 25$ in Fig. 16A, and $\left[\left(\mathrm{BaTiO}_{3}\right)_{8} /\left(\mathrm{SrTiO}_{3}\right)_{4}\right] \times 10$ and $\left[\left(\mathrm{BaTiO}_{3}\right)_{8} /\left(\mathrm{SrTiO}_{3}\right)_{4}\right] \times 40$ (strain partially relaxed) in Fig. 16B. Both $\mathrm{TO}_{2}$ and $\mathrm{TO}_{4}$ phonons show similar behaviors and the dashed-dotted lines are linear fits to the average of the two modes. The linear fit corresponds to a parabolic decrease of polarization with temperature as Raman intensity is proportional to the square of atomic displacement. The intersection of a dash-dotted line with the horizontal axis is taken as the $T_{c}$ of the sample.

The temperature dependence of polarization from a phase-field model calculation ${ }^{121}$ is plotted in Figs. 16C and 16D for the same samples as in Figs. 16A and B. The model assumes that the $\mathrm{BaTiO}_{3}$ and $\mathrm{SrTiO}_{3}$ layers in the superlattices have their respective bulk elastic and thermodynamic properties. The in-plane lattice constant is commensurately constrained to the $\mathrm{SrTiO}_{3}$ substrate except for the partially relaxed case, and the top surface is stress-free. The surface depolarization field is ignored and a short-circuit electrostatic boundary condition is employed. A computational cell of $64 \mathrm{~nm}$ along the two in-plane directions and one unit cell along the growth direction was employed. The corresponding 3-dimensional time- 
This is an author-produced, peer-reviewed version of this article. The final, definitive version of this document can be found online at Journal of the

American Ceramic Society, published by Blackwell Publishing. Copyright restrictions may apply. doi: 10.1111/j.1551-2916.2008.02371.x

dependent Ginzburg-Landau equations are then numerically solved using the perturbation method with semi-implicit Fourier-spectral algorithms ${ }^{122}$. The result reveals a spontaneous polarization along the growth direction with multiple $180^{\circ}$ domains in the $\mathrm{BaTiO}_{3}$ layers, which induces polarization in the adjacent $\mathrm{SrTiO}_{3}$ layers whose magnitude and distribution vary with the thickness and domain size of the $\mathrm{BaTiO}_{3}$ layers. The spontaneous polarization in the $\mathrm{BaTiO}_{3}$ layers becomes zero at $T_{c}$, and the predicted $T_{c}$ values agree with those from the Raman data. This is remarkable considering that no fitting parameters from the Raman experiments are used in the calculations.

In Fig. 17, $T_{c}$ determined by the Raman data, XRD, and the phase-field model are shown as a function of the $\mathrm{BaTiO}_{3}$ and $\mathrm{SrTiO}_{3}$ layer thicknesses. The XRD measurement provides an additional confirmation of the Raman results, where a change in the temperature dependence of the out-of-plane lattice constant can be taken as an indication of $T_{c}{ }^{116}$. The figure shows that the $\mathrm{BaTiO}_{3}$ layers in the superlattices are ferroelectric even when their thickness is only one unit cell, with a $T_{c}$ as high as $250 \mathrm{~K} . T_{c}$ increases with increasing $n$ as the dipole-dipole interaction in $\mathrm{BaTiO}_{3}$ layers becomes stronger, while large $m$ suppresses $T_{c}$ by reducing the coupling between the $\mathrm{BaTiO}_{3}$ layers. By changing the values of $n$ and $m$, we were able to tune $T_{c}$ from $151 \mathrm{~K}$ to $638 \mathrm{~K}$, i.e. from $250 \mathrm{~K}$ below to $235 \mathrm{~K}$ above the bulk value of $\mathrm{BaTiO}_{3}$. The higher-than-bulk $T_{c}$ is due to the strain in the $\mathrm{BaTiO}_{3}$ layers, just as strain enhances $T_{c}$ in single-layer ferroelectric films ${ }^{123,124}$. When the strain is partially relaxed in sample $\left(\mathrm{BTO}_{8} / \mathrm{STO}_{4}\right) \times 40, T_{c}$ drops to $440 \mathrm{~K}$, almost to the bulk $\mathrm{BaTiO}_{3}$ value. While the 3-dimensional phase-field model allowing domain formation provides a good description of the Raman data, simulations assuming a single domain in the $\mathrm{BaTiO}_{3}$ layers yield significantly lower $T_{c}$ for $m=13$, demonstrating the importance of domain formation in theoretical calculations. ${ }^{125}$

We now can conclude that ferroelectricity can be very strong in one-unit-cell thick $\mathrm{BaTiO}_{3}$ layers $\left(T_{c} \sim 250 \mathrm{~K}\right.$ for $\left.n / m=1 / 4\right)$. The electrical boundary condition plays a critical role. With the highly-polarizable $\mathrm{SrTiO}_{3}$ in contact with the $\mathrm{BaTiO}_{3}$ layers, the critical thickness is reduced to a single unit cell. Meanwhile, the mechanical boundary condition imposed by the $\mathrm{SrTiO}_{3}$ substrate leads to strain in the $\mathrm{BaTiO}_{3}$ layers and thus enhanced ferroelectricity. The interplay between the electrical and mechanical boundary conditions enables the tuning of $T_{c}$ by nearly $500 \mathrm{~K}$. 
This is an author-produced, peer-reviewed version of this article. The final, definitive version of this document can be found online at Journal of the

American Ceramic Society, published by Blackwell Publishing. Copyright restrictions may apply. doi: 10.1111/j.1551-2916.2008.02371.x

\section{SUMMARY}

We discussed the applications of Raman spectroscopy to the studies of lattice dynamics in $(\mathrm{Ba}, \mathrm{Sr}) \mathrm{TiO}_{3}$ single crystals, thin films and superlattices. Raman studies of $\mathrm{SrTiO}_{3}$ and $\mathrm{Ba}_{x} \mathrm{Sr}_{1-x} \mathrm{TiO}_{3}$ films revealed essential differences in the lattice dynamical properties of thin films and corresponding single crystals, such as forbidden first order Raman scattering in films of paraelectric material $\mathrm{SrTiO}_{3}$, and in $\mathrm{Ba}_{x} \mathrm{Sr}_{1-x} \mathrm{TiO}_{3}$ films well above the ferroelectric phase transition temperature in bulk; hardening of the soft phonon modes in films compared to crystals; significantly larger range of the soft mode overdamping in the $\mathrm{Ba}_{x} \mathrm{Sr}_{1-x} \mathrm{TiO}_{3}$ films. On the other hand, the lattice dynamics behavior in the $\mathrm{Ba}_{x} \mathrm{Sr}_{1-x} \mathrm{TiO}_{3}$ films is found similar to that of relaxor ferroelectrics. Analogously to the relaxors, the presence of polar nanoregions existing in the films at the temperatures corresponding to the paraelectric phase in the bulk explains the specific lattice-dynamical properties of $\mathrm{SrTiO}_{3} \mathrm{Ba}_{x} \mathrm{Sr}_{1-x} \mathrm{TiO}_{3}$ thin films.

The effect of strain on the phase transitions in epitaxial $\mathrm{BaTiO}_{3}$ films has been studied. Strain strongly affects the phase diagram in the films, suppressing the transitions between different ferroelectric phases (tetragonal-orthorhombic-rhombohedral), characteristic of bulk $\mathrm{BaTiO}_{3}$, and stabilizing a single ferroelectric phase (orthorhombic in the case of $\mathrm{BaTiO}_{3}$ films which are tensile strained due to the thermal expansion mismatch and tetragonal in the case of coherent lattice-mismatch induced strain). The thermodynamic phase-field model calculations of the thin film phase diagrams are in agreement with Raman spectroscopy results.

Ultraviolet Raman spectroscopy is proved to be an effective technique allowing the observation of phonons and studying phase transitions in nanoscale ferroelectric superlattices and ultrathin films. From UV Raman results it was found that coherently-strained $\mathrm{BaTiO}_{3}$ layer in $\mathrm{BaTiO}_{3} / \mathrm{SrTiO}_{3}$ superlattices is ferroelectric even when its thickness is only one unit cell. Depending on thickness of $\mathrm{BaTiO}_{3}$ layers and strain, the phase transition temperature can be for about 500 degrees, from $\sim 170 \mathrm{~K}$ to $\sim 650 \mathrm{~K}$. Comparison of Raman data are with the thermodynamic calculations of polarization in superlattices reveals essential role of 180 domains. These results demonstrate the great utility of UV Raman spectroscopy for studying nanoscale ferroelectrics and show an example of the dramatic modification of material properties in artificially engineered epitaxial heterostructures. 
* Supported in part by the Basic Energy Sciences Division, U.S. Department of Energy, under Grant No. DE-FG02-01ER45907, by National Science Foundation under Grant No. DMR0705127, and by Research corporation (Grant No.7134)

$\dagger$ Electronic address: dmitritenne@boisestate.edu

1 Thin film ferroelectric materials and devices, edited by R. Ramesh (Kluwer Academic Publishers, Boston 1997)

2 R. Ramesh, S. Aggarwal, and O. Auciello, "Science and technology of ferroelectric films and heterostructures for non-volatile ferroelectric memories," Mater. Sci. Eng. R 32, 191-236 (2001).

3 M. Dawber, K. M. Rabe, and J. F. Scott, "Physics of thin-film ferroelectric oxides," Rev. Mod. Phys. 77, 1083-1130 (2005).

4 N. Setter, D. Damjanovic, L. Eng, G. Fox, S. Gevorgian, S. Hong, A. Kingon, H. Kohlstedt, N. Y. Park, G. B. Stephenson, I. Stolitchnov, A. K. Taganstev, D. V. Taylor, T. Yamada, and S. Streiffer, "Ferroelectric thin films: Review of materials, properties, and applications," J. Appl. Phys. 100, 051606 46pp. (2006).

5 S. K. Streiffer, C. Basceri, C. B. Parker, S. E. Lash, and A. I. Kingon, "Ferroelectricity in thin films: The dielectric response of fiber-textured $\left(\mathrm{Ba}_{x} \mathrm{Sr}_{1-x}\right) \mathrm{Ti}_{1+y} \mathrm{O}_{3+z}$ thin films grown by chemical vapor deposition,” J. Appl. Phys. 86, 4565-4575 (1999).

6 D. G. Schlom, J. H. Hanei, J. Lettieri, C. D. Theis, W. Tian, J. C. Jiang, and X. Q. Pan, "Oxide nano-engineering using MBE," Mater. Sci. Eng. B 87, 282-291 (2001).

7 C. H. Ahn, K. M. Rabe, and J.-M. Triscone, "Ferroelectricity at the nanoscale: Local polarization in oxide thin films and heterostructures," Science 303, 488-491 (2004).

8 Nanoscale Phenomena in Ferroelectric Thin Films, edited by Seungbum Hong (Springer, Berlin, 2004)

9 M. E. Lines and A. M. Glass, Principles and Applications of Ferroelectrics and Related Materials (Oxford University Press, New York 1977).

10 W. Cochran, "Crystal stability and the theory of ferroelectricity," Adv. Phys. 9, 387-423 (1960).

11 G. Rupprecht, R. O. Bell, and B. D. Silverman, "Nonlinearity and microwave losses in cubic strontium-titanate," Phys. Rev. 123, 97-98 (1961).

12 V. L. Gurevich and A. K. Tagantsev, "Intrinsic dielectric loss in crystals," Adv. Phys. 40, 
719-767 (1991).

13 A. K. Tagantsev, "Phonon mechanisms of intrinsic dielectric loss in crystals," in Ferroelectric Ceramics, edited by N. Setter and E. L. Colla (Birkhäuser, Basel, 1993), p. 127.

14 Landolt-Börnstein, Numerical Data and Functional Relationships in Science and Technology, New Series, Group III, Vol. 16 (Springer, 1981), p. 416.

15 J. D. Axe, J. Harada, and G. Shirane, "Anomalous acoustic dispersion in centrosymmetric crystals with soft optic phonons," Phys. Rev. B 1, 1227-1234 (1970).

16 W. G. Stirling, "Neutron inelastic scattering study of the lattice dynamics of strontium titanate: harmonic models," J. Phys. C 5, 2711-2730 (1972).

17 G. Shirane, J. D. Axe, J. Harada, and A. Linz, "Inelastic neutron scattering from single-domain BaTiO,", Phys. Rev. B 2, 3651-3657 (1970).

18 G. Shirane, J. D. Axe, J. Harada, and J. P. Remeika, "Soft ferroelectric modes in lead titanate," Phys. Rev. B 2, 155-159 (1970).

19 I. Tomeno, Y. Ishii, Y. Tsunoda, and K. Oka, " Lattice dynamics of tetragonal $\mathrm{PbTiO}_{3}$ ", Phys. Rev. B 73, 064116, 10pp. (2006).

20 J. Hlinka, M. Kempa, J. Kulda, P. Bourges, A. Kania, and J. Petzelt, ” Lattice dynamics of ferroelectric $\mathrm{PbTiO}_{3}$ by inelastic neutron scattering," Phys. Rev. B 73, 140101(R) 4pp. (2006).

21 J. T. Last, "Infrared-absorption studies on barium titanate and related materials," Phys. Rev. 105, 1740-1750 (1957).

22 W. G. Spitzer, R. C. Miller, D. A. Kleinman, and L. E. Howarth, "Far infrared dielectric dispersion in $\mathrm{BaTiO}_{3}, \mathrm{SrTiO}_{3}$, and $\mathrm{TiO}_{2}$ ", Phys. Rev. 126, 1710-1721 (1962).

23 J. L. Servoin, Y. Luspin, and F. Gervais, "Infrared dispersion in $\mathrm{SrTiO}_{3}$ at high temperature," Phys. Rev. B 22, 5501-5506 (1980); J. L. Servoin, F. Gervais, A. M. Quittet, and Y. Luspin, "Infrared and Raman responses in ferroelectric perovskite crystals: Apparent inconsistencies," ibid. 21, 2038-2041 (1980).

24 C. H. Perry and D. B. Hall, "Temperature dependence of the Raman spectrum of $\mathrm{BaTiO}_{3}$ ", Phys. Rev. Lett. 15, 700-702 (1965).

25 M. DiDomenico, Jr., S. P. S. Porto, and S. H. Wemple, "Evidence from Raman scattering for an overdamped soft optic mode in $\mathrm{BaTiO}_{3}$ ", Phys. Rev. Lett. 19, 855-857 (1967); M. DiDomenico, Jr., S. H. Wemple, S. P. S. Porto, and R. P. Bauman, "Raman spectrum of single-domain $\mathrm{BaTiO}_{3} "$, Phys. Rev. 174, 522-530 (1968). 
26 A. Scalabrin, A. S. Chaves, D. S. Shim, and S. P. S. Porto, "Temperature dependence of the $\mathrm{A}_{1}$ and E optical phonons in $\mathrm{BaTiO}_{3} "$, Phys. Status Solidi B 79, 731-742 (1977).

27 G. Burns and F. H. Dacol, "Lattice modes in ferroelectric perovskites. III. Soft modes in $\mathrm{BaTiO}_{3} "$, Phys. Rev. B 18, 5750-5755 (1978).

28 H. Vogt, J. A. Sanjurjo, and G. Rossbroich, "Soft-mode spectroscopy in cubic $\mathrm{BaTiO}_{3}$ by hyper-Raman scattering," Phys. Rev. B 26, 5904-5910 (1982); H. Presting, J. A. Sanjurjo, and H. Vogt, "Mode softening in cubic $\mathrm{BaTiO}_{3}$ and the problem of its adequate description," ibid. 28, 6097-6099 (1983).

29 J. D. Freire and R. S. Katiyar, "Lattice dynamics of crystals with tetragonal $\mathrm{BaTiO}_{3}$ structure," Phys. Rev. B 37, 2074-2085 (1988).

30 U. D. Venkateswaran, V. M. Naik, and R. Naik, "High-pressure Raman studies of polycrystalline $\mathrm{BaTiO}_{3}$ ", Phys. Rev. B 58, 14256-14260 (1998).

31 M. Osada, M. Kakihana, S. Wada, T. Noma, and W.-S. Cho, "Broken symmetry in lowtemperature $\mathrm{BaTiO}_{3}$ phases: Strain effects probed by Raman scattering," Appl. Phys. Lett., 75, 3393-3395 (1999).

32 W. G. Nilsen and J. G. Skinner, "Raman spectrum of strontium titanate," J. Chem. Phys. 48, 2240-2248 (1968).

33 P. A. Fleury and J. M. Worlock, "Electric-Field-Induced Raman Scattering in $\mathrm{SrTiO}_{3}$ and $\mathrm{KTaO}_{3}$ ", Phys. Rev. 174, 613-623 (1968).

34 H. Vogt and G. Rossbroich, "Accurate determination of the far-infrared dispersion in $\mathrm{SrTiO}_{3}$ by hyper-Raman spectroscopy," Phys. Rev. B 24, 3086-3091 (1981); H. Vogt, "Hyper-Raman tensors of the zone-center optical phonons in $\mathrm{SrTiO}_{3}$ and $\mathrm{KTaO}_{3}$ ", ibid. 38, 5699-5708 (1988).

35 G. Burns and B. A. Scott, "Raman studies of underdamped soft modes in $\mathrm{PbTiO}_{3}$ ", Phys. Rev. Lett. 25, 167-170 (1970); "Lattice Modes in Ferroelectric Perovskites: $\mathrm{PbTiO}_{3}$ ", Phys. Rev. B 7, 3088-3101 (1973).

36 I. Taguchi, A. Pignolet, L. Wang, M. Proctor, F. Levy, and P. E. Schmid, "Raman scattering from $\mathrm{PbTiO}_{3}$ thin films prepared on silicon substrates by radio frequency sputtering and thermal treatment," J. Appl. Phys. 73, 394-399 (1993).

37 H. Zhang, S. Leppãvuori, P. Karjalainen, "Raman spectra in laser ablated lead zirconate titanate thin films near the morphotropic phase boundary," J. Appl. Phys. 77, 2691-2696 (1995). 
This is an author-produced, peer-reviewed version of this article. The final, definitive version of this document can be found online at Journal of the American Ceramic Society, published by Blackwell Publishing. Copyright restrictions may apply. doi: 10.1111/j.1551-2916.2008.02371.x

38 E. Ching-Prado, J. Cordero, R. S. Katiyar, and A. S. Bhalla, "Temperature-dependent Raman scattering in PT and PMN-PT thin films," J. Vac. Sci. Technol. A 14, 762-767 (1996).

39 Z. C. Feng, B. S. Kwak, A. Erbil, and L. A. Boatner, "Difference Raman spectra of $\mathrm{PbTiO}_{3}$ thin films grown by metalorganic chemical vapor deposition," Appl. Phys. Lett. 62, 349-351 (1993.

40 L. H. Robins, D. L. Kaiser, L. D. Rotter, P. K. Schenck, G. T. Stauf, and D. Rytz, "Investigation of the structure of barium titanate thin films by Raman spectroscopy," J. Appl. Phys. 76, 7487-7498 (1994).

41 Yu. I. Yuzyuk, V. A. Alyoshin, I. N. Zakharchenko, E. V. Sviridov, A. Almeida, and M. R. Chaves, "Polarization-dependent Raman spectra of heteroepitaxial (Ba,Sr) $\mathrm{TiO}_{3} / \mathrm{MgO}$ thin films," Phys. Rev. B 65, 134107, 9pp. (2002)

42 Yu. I. Yuzyuk, P. Simon, I. N. Zakharchenko, V. A. Alyoshin, and E. V. Sviridov, "Stress effect on the ferroelectric-to-paraelectric phase transition in heteroepitaxial $(\mathrm{Ba}, \mathrm{Sr}) \mathrm{TiO}_{3} /(001) \mathrm{MgO}$ thin film studied by Raman scattering and x-ray diffraction," Phys. Rev. B 66, 052103, 4pp. (2002).

43 Yu. I. Yuzyuk, J. L. Sauvajol, P. Simon, V. L. Lorman, V. A. Alyoshin, I. N. Zakharchenko, and E. V. Sviridov, "Phase transitions in $\left(\mathrm{Ba}_{0.7} \mathrm{Sr}_{0.3}\right) \mathrm{TiO}_{3} /(001) \mathrm{MgO}$ thin film studied by Raman scattering," J. Appl. Phys. 93, 9930-9937 (2003)

44 Yu. I. Yuzyuk, R. S. Katiyar, V. A. Alyoshin, I. N. Zakharchenko, D. A. Markov, and E. V. Sviridov, "Stress relaxation in heteroepitaxial $(\mathrm{Ba}, \mathrm{Sr}) \mathrm{TiO}_{3} /(001) \mathrm{MgO}$ thin film studied by micro-Raman spectroscopy" Phys. Rev. B 68, 104104, 4pp. (2003).

45 V. I. Merkulov, J. R. Fox, H.-C. Li, W. Si, A. A. Sirenko, and X. X. Xi, "Metal-oxide bilayer Raman scattering in $\mathrm{SrTiO}_{3}$ thin films," Appl. Phys. Lett. 72, 3291-3293 (1998).

46 A. A. Sirenko, I. A. Akimov, J. R. Fox, A. M. Clark, Hong-Cheng Li, Weidong Si, and X. X. $\mathrm{Xi}$, "Observation of the first-order Raman scattering in $\mathrm{SrTiO}_{3}$ thin films," Phys. Rev. Lett. 82, 4500-4503 (1999).

47 I. A. Akimov, A. A. Sirenko, A. M. Clark, J.-H. Hao, and X. X. Xi, "Electric-field-induced soft-mode hardening in $\mathrm{SrTiO}_{3}$ films," Phys. Rev. Lett. 84, 4625-4628 (2000)

48 D. A. Tenne, A. M. Clark, A. R. James, K. Chen, and X. X. Xi, "Soft phonon modes in $\mathrm{Ba}_{0.5} \mathrm{Sr}_{0.5} \mathrm{TiO}_{3}$ thin films studied by Raman spectroscopy," Appl. Phys. Lett. 79, 3836-3838 (2001). 
49 D. A. Tenne, A. Soukiassian, M. H. Zhu, A. M. Clark, X. X. Xi, H. Choosuwan, Qi He, R. Guo, and A. S. Bhalla, "Raman study of $\mathrm{Ba}_{x} \mathrm{Sr}_{1-x} \mathrm{TiO}_{3}$ films: evidence for the existence of polar nanoregions," Phys. Rev. B 67, 012302, 4pp. (2003).

50 D. A. Tenne, X. X. Xi, Y. L. Li, L. Q. Chen, A. Soukiassian, M. H. Zhu, A. R. James, J. Lettieri, D. G. Schlom, W. Tian, and X. Q. Pan, " Absence of low-temperature phase transitions in epitaxial $\mathrm{BaTiO}_{3}$ thin films," Phys. Rev. B 69, 174101, 5pp. (2004).

51 D. A. Tenne, A. Soukiassian, X. X. Xi, H. Choosuwan, R. Guo, and A. S. Bhalla, "Lattice dynamics in $\mathrm{Ba}_{x} \mathrm{Sr}_{1-x} \mathrm{TiO}_{3}$ thin films studied by Raman spectroscopy," J. Appl. Phys. 96, 6597-6605 (2004).

52 A. A. Sirenko, C. Bernhard, A. Golnik, Anna M. Clark, Jianhua Hao, Weidong Si, and X. X. Xi, "Soft-mode hardening in $\mathrm{SrTiO}_{3}$ thin films," Nature 404, 373-376 (2000).

53 D. A. Tenne, A. Bruchhausen, N. D. Lanzillotti-Kimura, A. Fainstein, R. S. Katiyar, A. Cantarero, A. Soukiassian, V. Vaithyanathan, J. H. Haeni, W. Tian, D. G. Schlom, K. J. Choi, D. M. Kim, C. B. Eom, H. P. Sun, X. Q. Pan, Y. L. Li, L. Q. Chen, Q. X. Jia, S. M. Nakhmanson, K. M. Rabe, X. X. Xi, "Probing nanoscale ferroelectricity by ultraviolet Raman spectroscopy," Science 313, 1614-1616 (2006)

54 V. V. Lemanov, E. P. Smirnova, P. P. Syrnikov, and E. A. Tarakanov, "Phase transitions and glasslike behavior in $\mathrm{Sr}_{1-x} \mathrm{Ba}_{x} \mathrm{TiO}_{3} "$, Phys. Rev. B 54, 3151-3157 (1996).

55 K. A. Müller and H. Burkard, "SrTiO 3 : an intrinsic quantum paraelectric below $4 \mathrm{~K}$," Phys. Rev. B 19, 3593-3602 (1979).

56 J. G. Bednorz and K. A. Müller, " $\mathrm{Sr}_{1-x} \mathrm{Ca}_{x} \mathrm{TiO}_{3}$ : An XY quantum ferroelectric with transition to randomness," Phys. Rev. Lett. 52, 2289-2292 (1984).

57 Ang Chen, Zhi Yu, J. Scott, A. Loidl, R. Guo, A. S. Bhalla and L. E. Cross, "Dielectric polarization processes in Bi:SrTiO3”, J. Phys. Chem. Solids 61, 191-196 (2000).

58 M. Itoh, R. Wang, Y. Inaguma, T. Yamaguchi, Y-J. Shan, and T. Nakamura, "Ferroelectricity induced by oxygen isotope exchange in strontium titanate perovskite," Phys. Rev. Lett. 82, 3540-3543 (1999).

59 H. Uwe and T. Sakudo, "Stress-induced ferroelectricity and soft phonon modes in $\mathrm{SrTiO}_{3}$ ", Phys. Rev. B 13, 271-286 (1976).

60 N. A. Pertsev, A. G. Zembilgotov, and A. K. Tagantsev, "Effect of mechanical boundary conditions on phase diagrams of epitaxial ferroelectric thin films," Phys. Rev. Lett. 80, 1988- 
1991 (1998).

61 N. A. Pertsev, A. K. Tagantsev, and N. Setter, "Phase transitions and strain-induced ferroelectricity in $\mathrm{SrTiO}_{3}$ epitaxial thin films," Phys. Rev. B 61, R825-R829 (2000).

${ }^{62}$ K. Laabidi, M. D. Fontana, and B. Jannot, "Underdamped soft phonon in orthorhombic $\mathrm{BaTiO}_{3}$ ", Solid State Commun. 76, 765-768 (1990).

63 J. Harada, J. D. Axe, and G. Shirane, "Neutron-scattering study of soft modes in cubic $\mathrm{BaTiO}_{3}$ ", Phys Rev. B 4, 155-162 (1971).

64 R. Comes, M. Lambert, and A. Guinier, "Chain structure of $\mathrm{BaTiO}_{3}$ and $\mathrm{KNbO}_{3}$ ", Solid State Commun. 6, 715-719 (1968); "Linear disorder in crystals (silicon, quartz and ferroelectric perovskites)," Acta Crystallogr. A 26, 244-254 (1970).

65 K. A. Müller and W. Berlinger, "Microscopic probing of order-disorder versus displacive behavior in $\mathrm{BaTiO}_{3}$ by Fe ${ }^{3+}$ EPR," Phys. Rev. B 34, 6130-6136 (1986).

66 R. E. Cohen and H. Krakauer, "Lattice dynamics and origin of ferroelectricity in $\mathrm{BaTiO}_{3}$ : Linearized-augmented-plane-wave total-energy calculations," Phys. Rev. B 42, 6416-6423 (1990).

67 J. P. Sokoloff, L. L. Chase, and D. Rytz, "Direct observation of relaxation modes in $\mathrm{KNbO}_{3}$ and $\mathrm{BaTiO}_{3}$ using inelastic light scattering," Phys. Rev. B 38, 597-605 (1988); "Reply to Comment on Direct observation of relaxation modes in $\mathrm{KNbO}_{3}$ and $\mathrm{BaTiO}_{3}$ using inelastic light scattering," ibid. 40, 788-790 (1989).

68 M. D. Fontana, K. Laabidi, and B. Jannot, "Quasimodes and a central peak in BaTiO 3 ", J. Phys. Condens. Matter 6, 8923-8930 (1994).

69 T. P. Dougherty, G. P. Wiederrecht, K. A. Nelson, M. H. Garrett, H. P. Jenssen, and C. Warde, "Femtosecond time-resolved spectroscopy of soft modes in structural phase transitions of perovskites," Phys. Rev. B 50, 8996-9019 (1994).

70 B. Zalar,V. V. Laguta, and R. Blinc, "NMR Evidence for the coexistence of order-disorder and displacive components in barium titanate," Phys. Rev. Lett. 90, 037601, 4pp. (2003).

71 M. Stachiotti, A. Dobry, R. Migoni, and A. Bussmann-Holder, "Crossover from a displacive to an order-disorder transition in the nonlinear-polarizability model," Phys. Rev. B 47, 2473-2479 (1993).

72 A. Bussmann-Holder, "Soft modes and order-disorder effects in ferroelectric phase transitions," J. Phys. Chem. Solids 57, 1445-1448 (1996). 
This is an author-produced, peer-reviewed version of this article. The final, definitive version of this document can be found online at Journal of the American Ceramic Society, published by Blackwell Publishing. Copyright restrictions may apply. doi: 10.1111/j.1551-2916.2008.02371.x

73 Y. Girshberg and Y. Yakobi, "Ferroelectric phase transitions and off-centre displacements in systems with strong electron-phonon interaction," J. Phys. Condens. Matter 11, 9807-9822 (1999).

74 H. J. Bakker, "Unified description of the soft and the relaxational mode in the dielectric response of ferroelectrics," Phys. Rev. B 52, 4093-4103 (1995).

75 W. Zhong, D. Vanderbilt, and K. M. Rabe, "Phase transitions in $\mathrm{BaTiO}_{3}$ from first principles," Phys. Rev. Lett. 73, 1861-1864 (1994); "First-principles theory of ferroelectric phase transitions for perovskites: The case of $\mathrm{BaTiO}_{3} "$, Phys. Rev. B 52, 6301-6312 (1995).

76 V. V. Lemanov, "Concentration dependence of phonon mode frequencies and the Grüneisen coefficients in $\mathrm{Ba}_{x} \mathrm{Sr}_{1-x} \mathrm{TiO}_{3}$ solid solutions," Phys. Solid State 39, 318-322 (1997).

77 S.-Y. Kuo, W.-Y. Liao, and W.-F. Hsieh, "Structural ordering transition and repulsion of the giant LO-TO splitting in polycrystalline $\mathrm{Ba}_{x} \mathrm{Sr}_{1-x} \mathrm{TiO}_{3}$ ", Phys. Rev. B 64, 224103, 7pp. (2001).

78 P. S. Dobal, A. Dixit, R. S. Katiyar, D. Garcia, R. Guo, and A. S. Bhalla, "Micro-Raman study of $\mathrm{Ba}_{1-x} \mathrm{Sr}_{x} \mathrm{TiO}_{3}$ ceramics," J. Raman Spectrosc. 32, 147-149 (2001).

79 R. Naik, J. J. Nazarko, C. S. Flattery, U. D. Venkateswaran, V. M. Naik, M. S. Mohammed, G. W. Auner, J. V. Mantese, N. W. Schubring, A. L. Micheli, and A. B. Catalan, "Temperature dependence of the Raman spectra of polycrystalline $\mathrm{Ba}_{1-x} \mathrm{Sr}_{x} \mathrm{TiO}_{3}$ ", Phys. Rev. B 61, 11367$11372(2000)$.

80 D. A. Tenne, A. Soukiassian, X. X. Xi, H. Choosuwan, R. Guo, and A. S. Bhalla, "Lattice dynamics in $\mathrm{Ba}_{x} \mathrm{Sr}_{1-x} \mathrm{TiO}_{3}$ single crystals: a Raman study," Phys. Rev. B 70, 174302, 9pp. (2004).

81 D. Garcia, R. Guo, and A. S. Bhalla, "Dielectric properties of $\mathrm{Ba}_{1-x} \mathrm{Sr}_{x} \mathrm{TiO}_{3}$ single crystal fibers grown by laser heated pedestal growth technique," Integr. Ferroelectrics 42, 57-69 (2002).

82 D. Kirillov, Y. Suzuki, L. Antognazza, K. Char, I. Bozovic, and T. H. Geballe, "Phonon anomalies at the magnetic phase transition in $\mathrm{SrRuO}_{3}$ ", Phys. Rev. B 51, 12825-12828 (1995).

83 H. Vogt, "Refined treatment of the model of linearly coupled anharmonic oscillators and its application to the temperature dependence of the zone-center soft-mode frequencies of $\mathrm{KTaO}_{3}$ and $\mathrm{SrTiO}_{3}, "$ Phys. Rev. B 51, 8046-8059 (1995).

84 J. Hemberger, P. Lunkenheimer, R. Viana, R. Bo"hmer, and A. Loidl, "Electric-field-dependent dielectric constant and nonlinear susceptibility in $\mathrm{SrTiO}_{3}$ ", Phys. Rev. B 52, 13159-13162 
(1995).

85 H. C. Li, W. Si, A. D. West, and X. X. Xi, "Near single crystal-level dielectric loss and nonlinearity in pulsed laser deposited $\mathrm{SrTiO}_{3}$ thin films," Appl. Phys. Lett. 73, 190-192 (1998).

86 J. Petzelt, T. Ostapchuk, I. Gregora, I. Rychetský, S. Hoffmann-Eifert, A. V. Pronin, Y. Yuzyuk, B. P. Gorshunov, S. Kamba, V. Bovtun, J. Pokorný, M. Savinov, V. Porokhonskyy, D. Rafaja, P. Vaněk, A. Almeida, M. R. Chaves, A. A. Volkov, M. Dressel, and R. Waser, "Dielectric, infrared, and Raman response of undoped $\mathrm{SrTiO}_{3}$ ceramics: Evidence of polar grain boundaries," Phys. Rev. B 64, 184111, 10pp. (2001).

87 J. Petzelt and T. Ostapchuk, "Soft-mode spectroscopy in $\mathrm{SrTiO}_{3}, \mathrm{BaTiO}_{3}$, and $\mathrm{BST}$ films and ceramics," Ferroelectrics 267, 93-100 (2002)

88 T. Ostapchuk, J. Petzelt, V. Železný, A. Pashkin, J. Pokorný, I. Drbohlav, R. Kuzěl, D. Rafaja, B. P. Gorshunov, M. Dressel, Ch. Ohly, S. Hoffmann-Eifert, and R. Waser, "Origin of soft-mode stiffening and reduced dielectric response in $\mathrm{SrTiO}_{3}$ thin films," Phys. Rev. B 66, 235406, 12pp. (2002).

89 T.-G. Kim, J. Oh, T. Moon, Y. Kim, B. Park, Y.-T. Lee, and S. Nam, "Effect of crystallinity on the dielectric loss of sputter-deposited $(\mathrm{Ba}, \mathrm{Sr}) \mathrm{TiO}_{3}$ thin films in the microwave range," J. Mater. Res. 18, 682-686 (2003).

90 P. M. Gehring, S. Wakimoto, Z.-G. Ye, and G. Shirane, "Soft Mode Dynamics above and below the Burns Temperature in the Relaxor $\mathrm{Pb}\left(\mathrm{Mg}_{1 / 3} \mathrm{Nb}_{2 / 3}\right) \mathrm{O}_{3}$ ", Phys. Rev. Lett. 87, 277601, 4pp. (2001).

91 S. Wakimoto, C. Stock, R. J. Birgeneau, Z.-G. Ye, W. Chen, W. J. L. Buyers, P. M. Gehring, and G. Shirane, "Ferroelectric ordering in the relaxor $\mathrm{Pb}\left(\mathrm{Mg}_{1 / 3} \mathrm{Nb}_{2 / 3}\right) \mathrm{O}_{3}$ as evidenced by low-temperature phonon anomalies" Phys. Rev. B 65, 172105, 4pp. (2002).

92 P. M. Gehring, S.-E. Park, and G. Shirane, "Soft Phonon Anomalies in the Relaxor Ferroelectric $\mathrm{Pb}\left(\mathrm{Zn}_{1 / 3} \mathrm{Nb}_{2 / 3}\right)_{0.92} \mathrm{Ti}_{0.08} \mathrm{O}_{3} "$, Phys. Rev. Lett. 84, 5216-5219 (2001).

93 T. Y. Koo, P. M. Gehring, G. Shirane, V. Kiryukhin, S.-G. Lee, and S-W. Cheong, "Anomalous transverse acoustic phonon broadening in the relaxor ferroelectric $\mathrm{Pb}\left(\mathrm{Mg}_{1 / 3} \mathrm{Nb}_{2 / 3}\right)_{0.8} \mathrm{Ti}_{0.2} \mathrm{O}_{3}$ ", Phys. Rev. B 65, 144113, 6pp. (2002).

94 L. E. Cross, "Relaxor ferroelectrics," Ferroelectrics 76, 241-267 (1987); "Relaxor ferroelectrics: an overview," ibid. 151, 305-320 (1994).

95 G. Burns and F. H. Dacol, "Crystalline ferroelectrics with glassy polarization behavior," 
This is an author-produced, peer-reviewed version of this article. The final, definitive version of this document can be found online at Journal of the

American Ceramic Society, published by Blackwell Publishing. Copyright restrictions may apply. doi: 10.1111/j.1551-2916.2008.02371.x

Phys. Rev. B 28, 2527-2530 (1983); "Glassy polarization behavior in ferroelectric compounds $\mathrm{Pb}\left(\mathrm{Mg}_{1 / 3} \mathrm{Nb}_{2 / 3}\right) \mathrm{O}_{3}$ and $\mathrm{Pb}\left(\mathrm{Zn}_{1 / 3} \mathrm{Nb}_{2 / 3}\right) \mathrm{O}_{3}$ ", Solid State Commun. 48, 853-856 (1983).

D. Viehland, S. J. Jang, L. E. Cross, and M. Wuttig, "Deviation from Curie-Weiss behavior in relaxor ferroelectrics," Phys. Rev. B 46, 8003-8006 (1992).

97 J. Toulouse, P. DiAntonio, B. E. Vugmeister, X. M. Wang, and L. A. Knauss, "Precursor effects and ferroelectric macroregions in $\mathrm{KTa}_{1-x} \mathrm{Nb}_{x} \mathrm{O}_{3}$ and $\mathrm{K}_{1-y} \mathrm{Li}_{y} \mathrm{TaO}_{3}$ ", Phys. Rev. Lett. 68, 232-235 (1992); P. DiAntonio, B. E. Vugmeister, J. Toulouse, and L. A. Boatner, "Polar fluctuations and first-order Raman scattering in highly polarizable $\mathrm{KTaO}_{3}$ crystals with offcenter Li and Nb" Phys. Rev. B, 47, 5629-5637 (1993).

98 H. Vogt, "Hyper-Raman study of Li-induced polarization clusters in $\mathrm{K}_{1-x} \mathrm{Li}_{x} \mathrm{TaO}_{3}$ ", J. Phys. Condens. Matter 7, 5913-5929 (1995).

99 C. Hubert, J. Levy, T. V. Rivkin, C. Carlson, P. A. Parilla, J. D. Perkins, and D. S. Ginley, "Nanopolar reorientation in ferroelectric thin films," Appl. Phys. Lett. 79, 2058-2060 (2001).

100 C. Hubert, J. Levy, E. J. Cukauskas, and S. W. Kirchoefer, "Mesoscopic Microwave dispersion in ferroelectric thin films," Phys. Rev. Lett. 85, 1998-2001 (2000).

101 J. C. Wu, C. L. Jia, K. Urban, J. H. Hao, and X. X. Xi, "Microstructure and misfit relaxation in $\mathrm{SrTiO}_{3} / \mathrm{SrRuO}_{3}$ bilayer films on $\mathrm{LaAlO}_{3}(100)$ substrates," J. Mater. Researsh 16, 3443-3450 (2001).

102 R. Waser and D. M. Smyth, "Defect chemistry, conduction, and breakdown mechanism of perovskite-structure titanates," pp.47-92 in Ferroelectric Thin Films: Synthesis and Basic Properties, edited by C. P. de Araujo, J. F. Scott and G. W. Taylor (Gordon and Breach, Amsterdam, 1996).

103 H. Uwe, H. Yamaguchi, and T. Sakudo, "Ferroelectric microregion in $\mathrm{KTa}_{1-x} \mathrm{Nb}_{x} \mathrm{O}_{3}$ and $\mathrm{SrTiO}_{3}$ ", Ferroelectrics 96, 123-126 (1989).

104 D. A. Crandles, B. Nicholas, C. Dreher, C. C. Homes, A. W. McConnell, B. P. Clayman, W. H. Gong, and J. E. Greedan, "Optical properties of highly reduced $\mathrm{SrTiO}_{3-x}$ ", Phys. Rev. B 59, 12842-12846 (1999).

105 D. A. Tenne, I. E. Gonenli, A. Soukiassian, D. G. Schlom, S. M. Nakhmanson, K. M. Rabe, and X. X. Xi, "Raman study of oxygen reduced and re-oxidized strontium titanate," Phys. Rev. B 76, 024303, 7pp. (2007).

106 U. Bianchi, W. Kleemann, and J. G. Bednorz, "Raman scattering of ferroelectric 
$\mathrm{Sr}_{1-x} \mathrm{Ca}_{x} \mathrm{TiO}_{3}, \mathrm{x}=0.007$ ", J. Phys. Condens. Matter 6, 1229-1238 (1994).

107 V. Porokhonskyy, A. Pashkin, V. Bovtun, J. Petzelt, M. Savinov, P. Samoukhina, T. Ostapchuk, J. Pokorný, M. Avdeev, A. Kholkin, and P. Vilarinho, " Broad-band dielectric spectroscopy of $\mathrm{SrTiO}_{3}$ :Bi ceramics," Phys. Rev. B 69, 144104, 10pp. (2004).

D. G. Schlom, L.-Q. Chen, C.-B. Eom, K. M. Rabe, S. K. Streiffer, and J.-Marc Triscone, "Strain Tuning of Ferroelectric Thin Films," Annu. Rev. Mater. Res. 37, 589-626 (2007), and references therein.

109 Y. L. Li, S. Choudhury, J. H. Haeni, M. D. Biegalski, A. Vasudevarao, A. Sharan, H. Z. Ma, J. Levy, V. Gopalan, S. Trolier-McKinstry, D. G. Schlom, Q. X. Jia, and L. Q. Chen, "Phase transitions and domain structures in strained pseudocubic (100) $\mathrm{SrTiO}_{3}$ thin films," Phys. Rev. B 73, 184112, 13pp. (2006).

110 D. Taylor, "Thermal Expansion Data VIII. Complex Oxides, $\mathrm{ABO}_{3}$, the Perovskites," Transactions and Journal of the British Ceramic Society 84, 181-188 (1985).

111 J. J. Neumeier, A. L. Cornelius, and K. Andres, "Thermodynamic investigation of the magnetic phase transitions of $\mathrm{CaMnO}_{3}$ and $\mathrm{SrRuO}_{3}$ ", Phys. Rev. B 64, 172406, 3pp. (2001).

112 Y. L. Li and L.-Q. Chen, "Temperature-strain phase diagram for $\mathrm{BaTiO}_{3}$ thin films," Appl. Phys. Lett. 88, 072905, 3pp. 2006.

113 R. R. Das, Yu. I. Yuzyuk, P. Bhattacharya, V. Gupta, and R. S. Katiyar, "Folded acoustic phonons and soft mode dynamics in $\mathrm{BaTiO}_{3} / \mathrm{SrTiO}_{3}$ superlattices," Phys. Rev. B 69, 132302, 4pp. (2004).

114 R. S. Katiyar, Y. I. Yuzyuk, R. R. Das, P. Bhattacharya, V. Gupta, "Polarized Raman spectra of $\mathrm{BaTiO}_{3} / \mathrm{SrTiO}_{3}$ superlattices," Ferroelectrics 329, 907-916 (2005).

115 L. Hilt Tisinger, R. Liu, J. Kulik, X. Zhang, J. Ramdani, and A. A. Demkov, "UltravioletRaman studies of $\mathrm{SrTiO}_{3}$ ultrathin films on Si," J. Vac. Sci. Technol. B 21 53-56 (2003).

116 see Supporting Online Material for Ref. 53.

117 B. Jusserand and M. Cardona, "Raman spectroscopy of vibrations in superlattices," pp. 49152 in Light Scattering in Solids V, edited by M. Cardona and G. Güntherodt (Springer, Heidelberg, 1989).

118 C. Colvard, T. A. Gant, M. V. Klein, R. Merlin, R. Fischer, H. Morkoc, and A. C. Gossard, "Folded acoustic and quantized optic phonons in (GaAl)As superlattices," Phys. Rev. B 31, 2080-2091 (1985). 
119 M. Trigo, A. Bruchhausen, A. Fainstein, B. Jusserand, V. Thierry-Mieg, "Confinement of acoustical vibrations in a semiconductor planar phonon cavity," Phys. Rev. Lett. 89, 227402, 4pp. (2002).

120 A. Bartels, T. Dekorsy, H. Kurz, K. Köhler, "Coherent zone-folded longitudinal acoustic phonons in semiconductor superlattices: excitation and detection," Phys. Rev. Lett. 82, 10441047 (1999).

121 L.-Q. Chen, "Phase-field models for microstructure evolution," Annu. Rev. Mater. Res. 32, 113-140 (2002).

122 L.-Q. Chen and J. Shen, "Applications of semi-implicit Fourier-spectral method to phase field equations," Comput. Phys. Commun. 108, 147-158 (1998).

123 K. J. Choi, M. Biegalski, Y. L. Li, A. Sharan, J. Schubert, R. Uecker, P. Reiche, Y. B. Chen, X. Q. Pan, V. Gopalan, L.-Q. Chen, D. G. Schlom, and C. B. Eom, "Enhancement of ferroelectricity in strained $\mathrm{BaTiO}_{3}$ thin films," Science 306, 1005-1009 (2004).

124 J. H. Haeni, P. Irvin, W. Chang, R. Uecker, P. Reiche, Y. L. Li, S. Choudhury, W. Tian, M. E. Hawley, B. Craigo, A. K. Tagantsev, X. Q. Pan, S. K. Streiffer, L. Q. Chen, S. W. Kirchoefer, J. Levy, and D. G. Schlom, "Room-temperature ferroelectricity in strained $\mathrm{SrTiO}_{3}$ ", Nature 430, 758-761 (2004).

125 V. A. Stephanovich, I. A. Luk'yanchuk, M. G. Karkut, ”omain-Enhanced Interlayer Coupling in Ferroelectric/Paraelectric Superlattices," Phys. Rev. Lett. 94, 047601, 4pp. (2005).

126 Y. L. Li, S. Y. Hu, D. Tenne, A. Soukiassian, D. G. Schlom, X. X. Xi, K. J. Choi, C. B. Eom, A. Saxena, T. Lookman, Q. X. Jia, and L.-Q. Chen, "Prediction of ferroelectricity in BaTiO3/SrTiO3 superlattices with domains," Appl. Phys. Lett. 91, 112914, 3pp. 2007. 


\section{FIGURE CAPTIONS}

FIG. 1: (a) Temperature evolution of Raman spectra of $\mathrm{Ba}_{0.1} \mathrm{Sr}_{0.9} \mathrm{TiO}_{3}$ crystal. Dashed-dotted lines indicate the soft modes. The peaks labelled $R$ in the spectra of $\mathrm{SrTiO}_{3}$ crystals are the structural modes. Vertical dashed-dotted lines are guides to eye. (b) Temperature dependence of the $E$ and $A_{1}$ soft mode frequencies (left and right panels, respectively) for $\mathrm{Ba}_{x} \mathrm{Sr}_{1-x} \mathrm{TiO}_{3}$ crystals with $\mathrm{x}=0.05,0.1,0.2$, and 0.5 . (After Tenne et al. ${ }^{80}$ )

FIG. 2: Composition dependence of the hard phonon mode frequencies at $5 \mathrm{~K}$. Dotted lines are guides to eye. The half-solid circles label the $T O_{2}$ and $L O_{1}$ modes, undistinguishable in spectra. (After Tenne et $a l .{ }^{80}$ )

FIG. 3: Raman spectra of $\mathrm{SrTiO}_{3}$ films grown on $\mathrm{SrRuO}_{3}$ buffer layers, and a single $\mathrm{SrTiO}_{3}$ crystal measured at $\mathrm{T}=5 \mathrm{~K}$ with $514.5 \mathrm{~nm}$ excitation. The peaks labelled $T O_{m}$ are the $\mathrm{TO}$ phonons of $\mathrm{SrTiO}_{3}, R$ - the structural modes due to antiferodistortive transition; the stars denote the $\mathrm{SrRuO}_{3}$ Raman lines. (After Sirenko et al. ${ }^{46}$ )

FIG. 4: (a) The square of the $\mathrm{TO}_{1}$ soft phonon frequency vs. temperature for a $2 \mu$ m-thick $\mathrm{SrTiO}_{3}$ film and a $\mathrm{SrTiO}_{3}$ single crystal. (b) The inverse dielectric constant vs. temperature for the same $\mathrm{SrTiO}_{3}$ film and single crystal. (After Sirenko et al. ${ }^{52}$ )

FIG. 5: Raman spectra of $1 \mu \mathrm{m}$-thick $\mathrm{SrTiO}_{3}$ film at $\mathrm{T}=5 \mathrm{~K}$ without electric field (solid line) and in the presence of an external electric field of $223104 \mathrm{~V} / \mathrm{cm}$ directed normal to the film plane (dotted line). The soft mode components are labelled $A$ and $E$. Structural modes are denoted by $R$, the $\mathrm{SrRuO}_{3}$ Raman lines - by the stars. (After Akimov et al. ${ }^{47}$ )

FIG. 6: Frequency of the $\mathrm{TO}_{1}$ phonon in $\mathrm{SrTiO}_{3}$ film as a function of temperature for different values of external electric field. Zero-field values of the $A$ mode are obtained by extrapolation and shown with open symbols. The dotted lines guide the eye. Electric-field values are given in $10^{4} \mathrm{~V} / \mathrm{cm}$. The vertical dashed line indicates the structural cubic-to-tetragonal phase transition observed in the film at $120 \mathrm{~K}$. (After Akimov et al. ${ }^{47}$ ) 
FIG. 7: Raman spectra of $\mathrm{Ba}_{0.2} \mathrm{Sr}_{0.8} \mathrm{TiO}_{3}$ film (top panel) and single crystal of the same composition (bottom panel) at 10 and $200 \mathrm{~K}$. In the top panel solid and dotted lines correspond to parallel and perpendicular polarization geometries, respectively. Stars mark the lines of $\mathrm{SrRuO}_{3}$ buffer layer. Vertical dashed-dotted lines are guides to eye. (After Tenne et al. ${ }^{49}$ )

FIG. 8: Temperature dependence of the $E$ and $F_{1 u}$ soft mode frequencies (circles) and damping (diamonds) for $\mathrm{Ba}_{0.2} \mathrm{Sr}_{0.8} \mathrm{TiO}_{3}$. Open symbols: single crystal, solid symbols: thin film. Horizontal bars show the temperature range where the soft mode is overdamped. (After Tenne et al. ${ }^{51}$ )

FIG. 9: The ranges of the $E$ soft mode overdamping as a function of Ba contents $x$ for $\mathrm{Ba}_{x} \mathrm{Sr}_{1-x} \mathrm{TiO}_{3}$ films (vertical error bars) and single crystals (shaded area). Symbols show the ferroelectric phase transition temperatures for single crystals (open circles) and films (triangles), as determined from the $A_{1}$ soft mode behavior. Dotted lines represent the phase diagram for bulk $\mathrm{Ba}_{x} \mathrm{Sr}_{1-x} \mathrm{TiO}_{3} .{ }^{54}$ (After Tenne et al. ${ }^{49}$ )

FIG. 10: (a) The inverse dielectric constant as a function of temperature for $\mathrm{Ba}_{0.2} \mathrm{Sr}_{0.8} \mathrm{TiO}_{3}$ film. (b) The temperature dependence of the square of the $E$ or $F_{1 u}$ soft mode frequency for the same film; dotted line shows the region where the $E$ soft mode is overdamped. (c) The $E$ or $F_{1 u}$ soft mode damping. Vertical dashed-dotted lines are guides to eye. (After Tenne et al. ${ }^{51}$ )

FIG. 11: Temperature evolution of Raman spectra of (a) a $\mathrm{BaTiO}_{3}$ single crystal and (b) a $\mathrm{BaTiO}_{3}$ film grown on $\mathrm{SrTiO}_{3}$ substrate with a $\mathrm{SrRuO}_{3}$ buffer layer, measured in parallel polarization geometry $z(x, x) \bar{z}$. Dashed-dotted lines are guides to eye. Star in the spectra of the film indicates features due to $\mathrm{SrRuO}_{3}$ layer. (After Tenne et al. ${ }^{50}$ )

FIG. 12: Temperature dependence of the $A_{1}\left(\mathrm{TO}_{2}\right)$ and $A_{1}\left(\mathrm{TO}_{4}\right)$ phonon frequencies for $\mathrm{BaTiO}_{3}$ single crystal (solid symbols) and film grown on $\mathrm{SrTiO}_{3}$ substrate with $\mathrm{SrRuO}_{3}$ buffer layer (open symbols). (After Tenne et al..$^{50}$ ) 
This is an author-produced, peer-reviewed version of this article. The final, definitive version of this document can be found online at Journal of the American Ceramic Society, published by Blackwell Publishing. Copyright restrictions may apply. doi: 10.1111/j.1551-2916.2008.02371.x

FIG. 13: Advantages of UV Raman spectroscopy for ferroelectric thin films over visible Raman. a): Schematic of the band structure, light absorption, and penetration depth of light in $\mathrm{SrTiO}_{3}$ ? as compared to the energies of the visible and UV photons. Strong absorption, small penetration depth, and strong resonance enhancement make UV Raman spectroscopy ideal for studying very thin ferroelectric films. b): spectra of (1) a bare $\mathrm{SrTiO}_{3}$ substrate (black curve), (2) a $\left.\left[\left(\mathrm{BaTiO}_{3}\right)_{5} /\left(\mathrm{SrTiO}_{3}\right)_{4}\right)\right] \times 25$ superlattice (green curve) measured with visible excitation $(514.5 \mathrm{~nm})$, and (3) the same superlattice measured with $351.1 \mathrm{~nm}$ UV excitation (purple curve). The dashed line shows the bare $\mathrm{SrTiO}_{3}$ substrate spectrum measured with $351.1 \mathrm{~nm}$ UV excitation. Triangles show the calculated frequencies of the first-order folded LA doublet. (After Tenne et al. ${ }^{53}$ ) $\mathbf{c}$ ): spectra of (1) $\left.\left[\left(\mathrm{BaTiO}_{3}\right)_{5} /\left(\mathrm{SrTiO}_{3}\right)_{4}\right)\right] \times 5$ superlattice, (2) and (3) - $\mathrm{BaTiO}_{3}$ films with thicknesses of 10 and $2.4 \mathrm{~nm}$, respectively, grown on $\mathrm{SrTiO}_{3}$, and (4) - a bare $\mathrm{SrTiO}_{3}$ substrate. The spectrum 1 is measured with $351.1 \mathrm{~nm}$ UV excitation, spectra 2-4 - with $325 \mathrm{~nm}$. Arrows mark the phonon peaks of the superlattice and the films.

FIG. 14: Raman spectra of the $\left[\left(\mathrm{BaTiO}_{3}\right)_{5} /\left(\mathrm{SrTiO}_{3}\right)_{4}\right] \times 25$ and $\left[\left(\mathrm{BaTiO}_{3}\right)_{8} /\left(\mathrm{SrTiO}_{3}\right)_{4}\right] \times 10$ superlattices at $295 \mathrm{~K}$, measured with $351.1 \mathrm{~nm}$ laser line. The spectra of $\mathrm{BaTiO}_{3}$ single crystals at 295, 190, and $100 \mathrm{~K}$, corresponding to tetragonal, orthorhombic, and rhombohedral phases, respectively, and the spectra of $\mathrm{SrTiO}_{3}$ single crystals at 295 and $5 \mathrm{~K}$ are shown for comparison. The bulk spectra were measured with the $514.5 \mathrm{~nm}$ laser line. (After Tenne et al. ${ }^{116}$ )

FIG. 15: Temperature evolution of UV Raman spectra of superlattices A $\left[\left(\mathrm{BaTiO}_{3}\right)_{5} /\left(\mathrm{SrTiO}_{3}\right)_{4}\right] \times 25$ and $\mathbf{B}\left[\left(\mathrm{BaTiO}_{3}\right)_{8} /\left(\mathrm{SrTiO}_{3}\right)_{4}\right] \times 10$. The arrows mark the $\mathrm{SrTiO}_{3}$-like $\mathrm{TO}_{2}$ mode at $180 \mathrm{~cm}^{-1}$ and the $\mathrm{TO}_{4}$ mode at about $530 \mathrm{~cm}^{-1}$, whose intensities decrease as the temperature increases and disappear at $T_{c}$.

FIG. 16: Temperature dependencies of normalized Raman intensities of $\mathrm{TO}_{2}$ (solid triangles) and $\mathrm{TO}_{4}$ (open triangles) phonons for $\mathbf{A}\left[\left(\mathrm{BaTiO}_{3}\right)_{2} /\left(\mathrm{SrTiO}_{3}\right)_{4}\right] \times 40$ and $\left[\left(\mathrm{BaTiO}_{3}\right)_{5} /\left(\mathrm{SrTiO}_{3}\right)_{4}\right] \times 25$ and $\mathbf{B}\left[\left(\mathrm{BaTiO}_{3}\right)_{8} /\left(\mathrm{SrTiO}_{3}\right)_{4}\right] \times 10$ and $\left[\left(\mathrm{BaTiO}_{3}\right)_{8} /\left(\mathrm{SrTiO}_{3}\right)_{4}\right] \times 40$. Sample $\left[\left(\mathrm{BaTiO}_{3}\right)_{8} /\left(\mathrm{SrTiO}_{3}\right)_{4}\right] \times 40$ is partially relaxed while the other three samples are commensurate with the $\mathrm{SrTiO}_{3}$ substrate. The dash-dotted lines are fits to a linear temperature dependence. $\mathbf{C}$ and $\mathbf{D}$ : The 3-dimensional phase-field model calculations of polarization as a function of temperature in the same superlattice samples. Polarization is given as a fraction of the polarization of bulk $\mathrm{BaTiO}_{3}, P_{0}=0.26 \mathrm{C} / \mathrm{m}^{2}$. (After Tenne et al. ${ }^{53}$ ) 
FIG. 17: Dependence of $T_{c}$ on $n$ and $m$ in superlattices $\left[\left(\mathrm{BaTiO}_{3}\right)_{n} /\left(\mathrm{SrTiO}_{3}\right)_{m}\right] \times N$, where the number of periods $N$ varies between 10 and 50. Blue symbols are for $m=4$ and red symbols for $m$ = 13. Open squares are from temperature-dependent XRD measurement. Solid lines are from the 3-dimensional phase-field model calculations, dashed lines - simulations assuming a single domain in the $\mathrm{BaTiO}_{3}$ layers. The black dash-dotted line shows the $T_{c}$ in bulk $\mathrm{BaTiO}_{3}$. (After $\mathrm{Li}$ et al. ${ }^{126}$ ) 
This is an author-produced, peer-reviewed version of this article. The final, definitive version of this document can be found online at Journal of the American Ceramic Society, published by Blackwell Publishing. Copyright restrictions may apply. doi: 10.1111/j.1551-2916.2008.02371.x

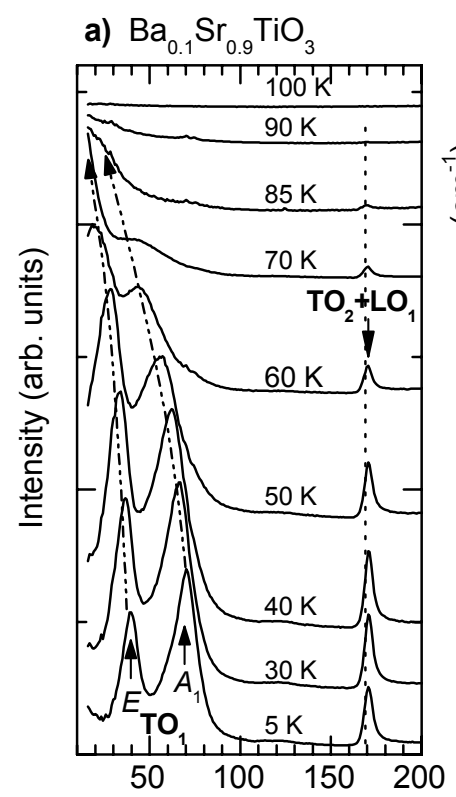

Raman shift $\left(\mathrm{cm}^{-1}\right)$ b) $E$ mode

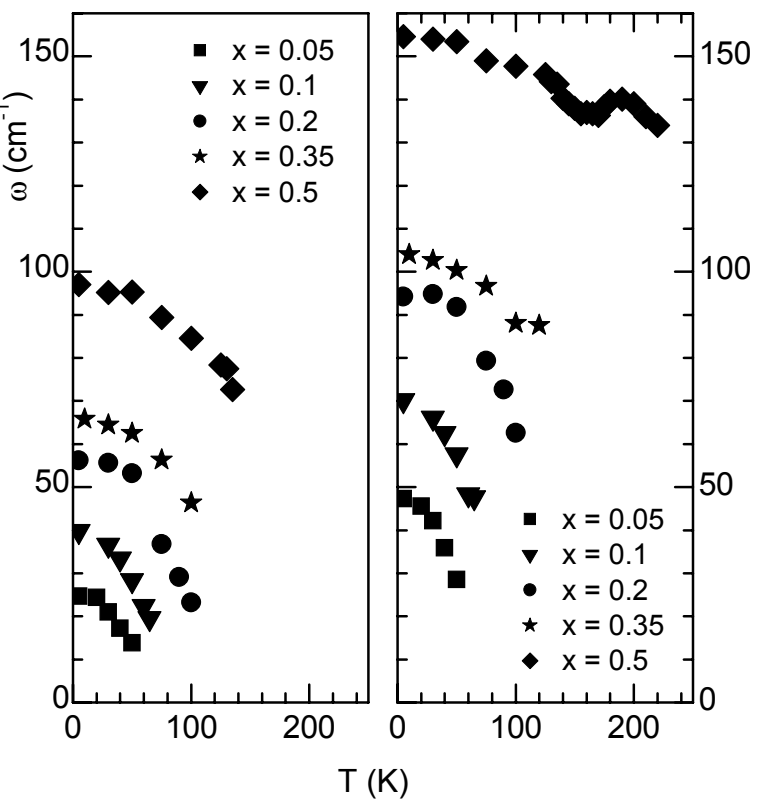

Figure 1

D. A. Tenne and X. X. Xi 
This is an author-produced, peer-reviewed version of this article. The final, definitive version of this document can be found online at Journal of the

American Ceramic Society, published by Blackwell Publishing. Copyright restrictions may apply. doi: 10.1111/j.1551-2916.2008.02371.x

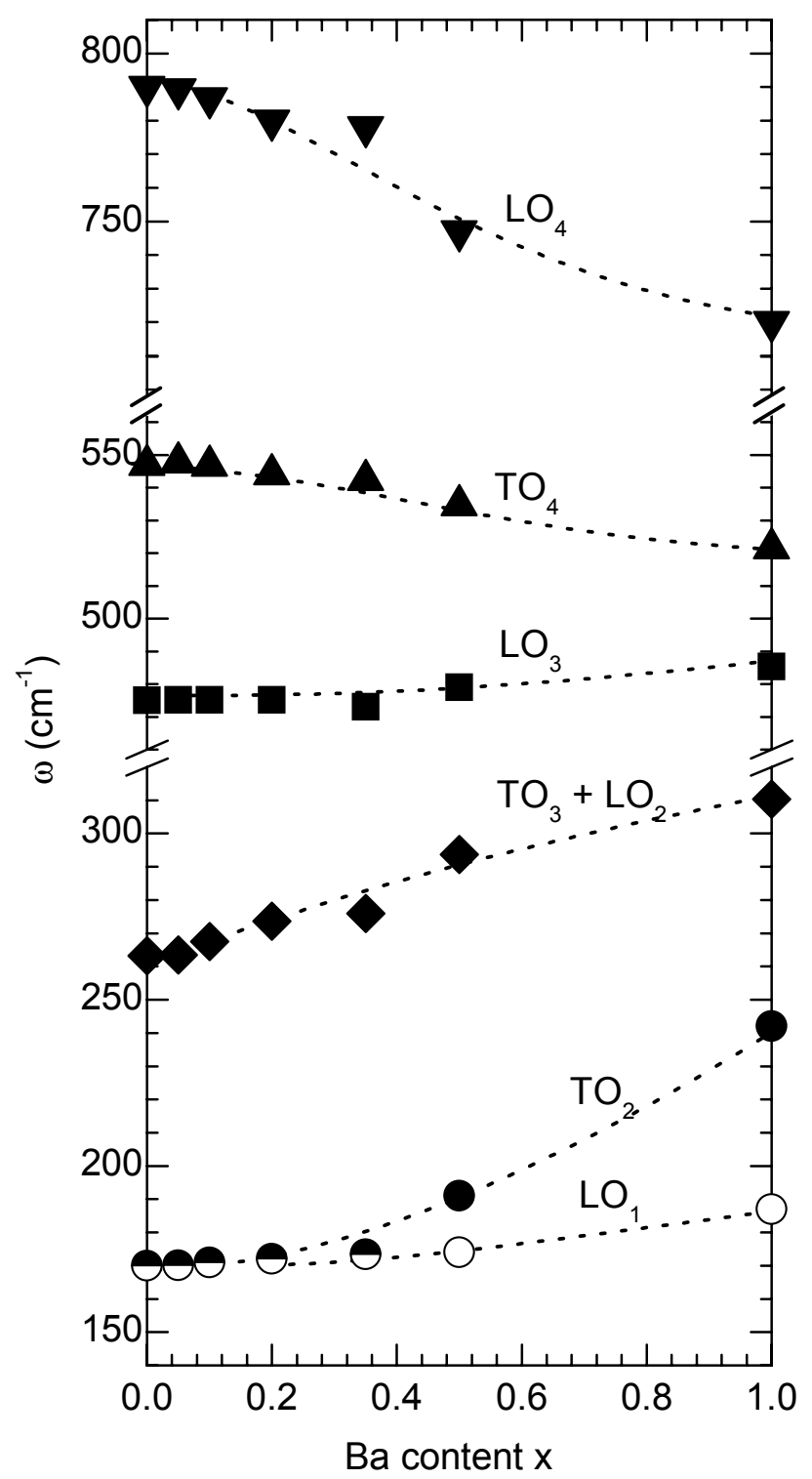

Figure 2

D. A. Tenne and X. X. Xi 


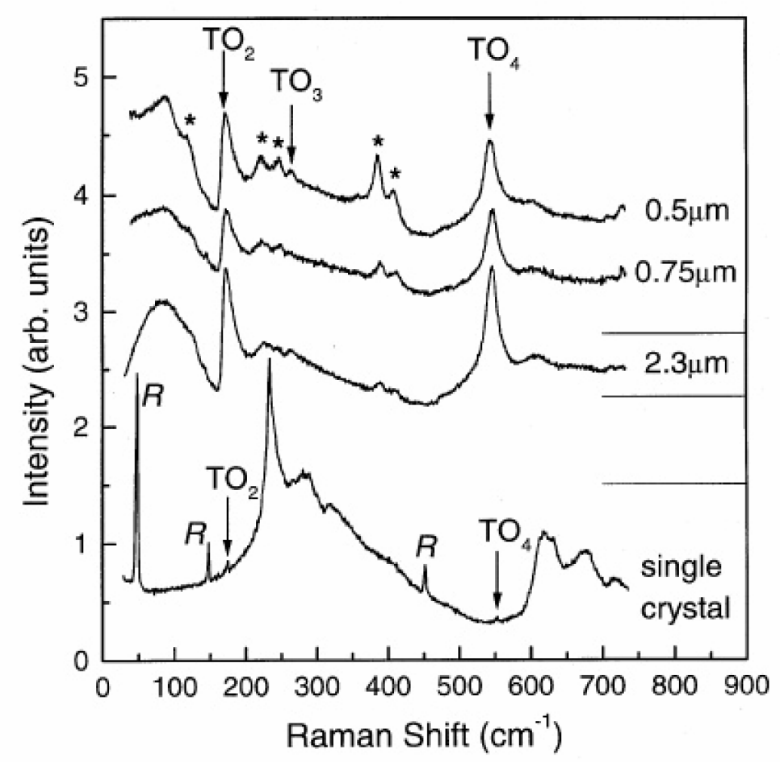

Figure 3

D. A. Tenne and X. X. Xi 


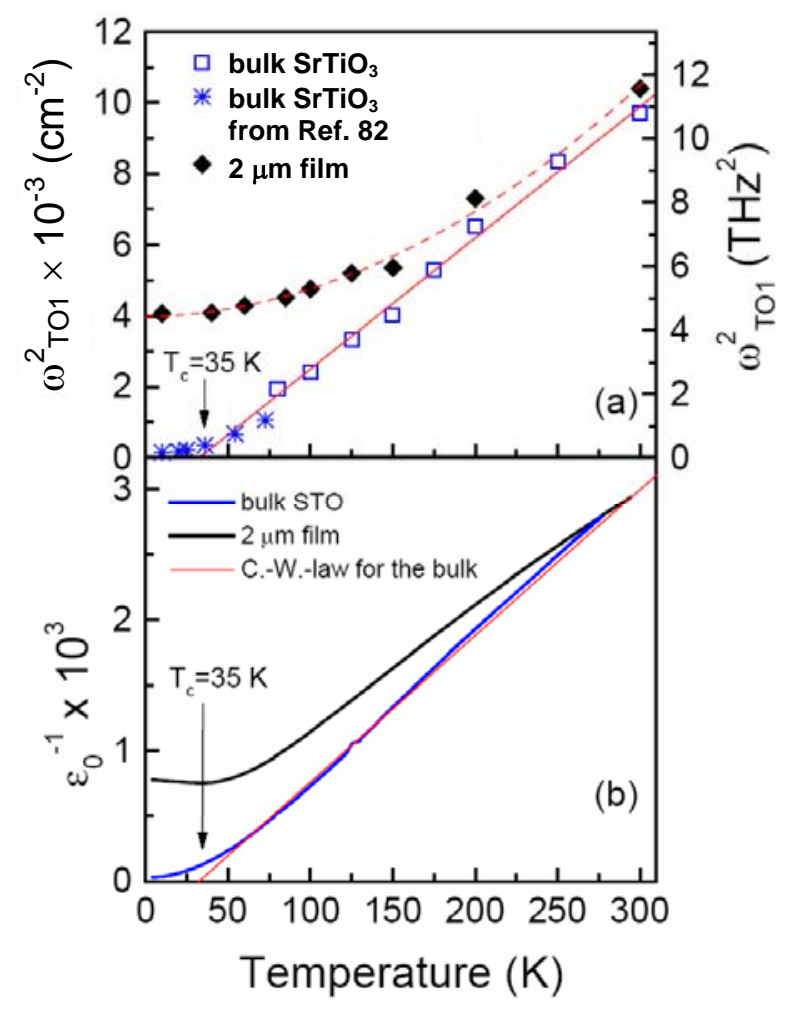

Figure 4

D. A. Tenne and X. X. Xi 


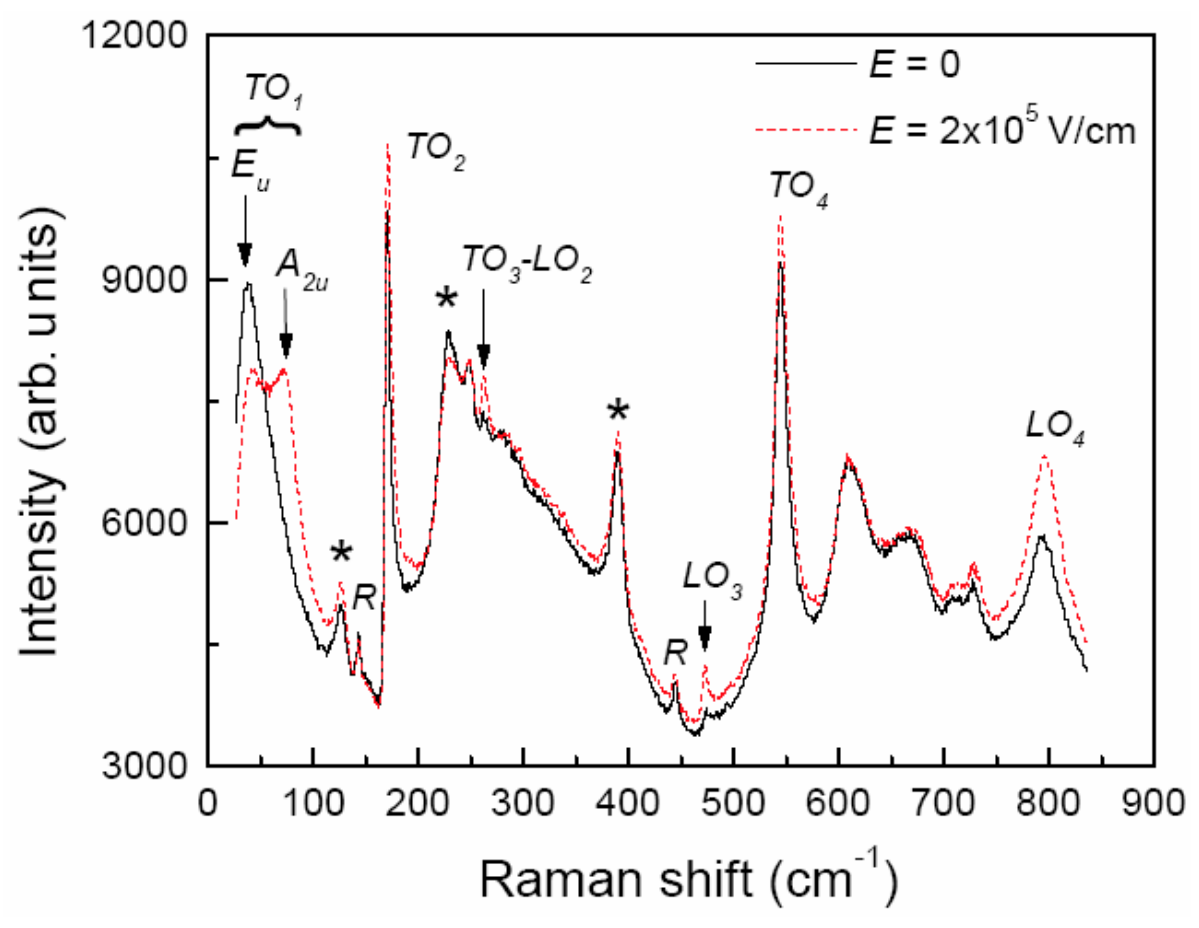

Figure 5

D. A. Tenne and X. X. Xi 


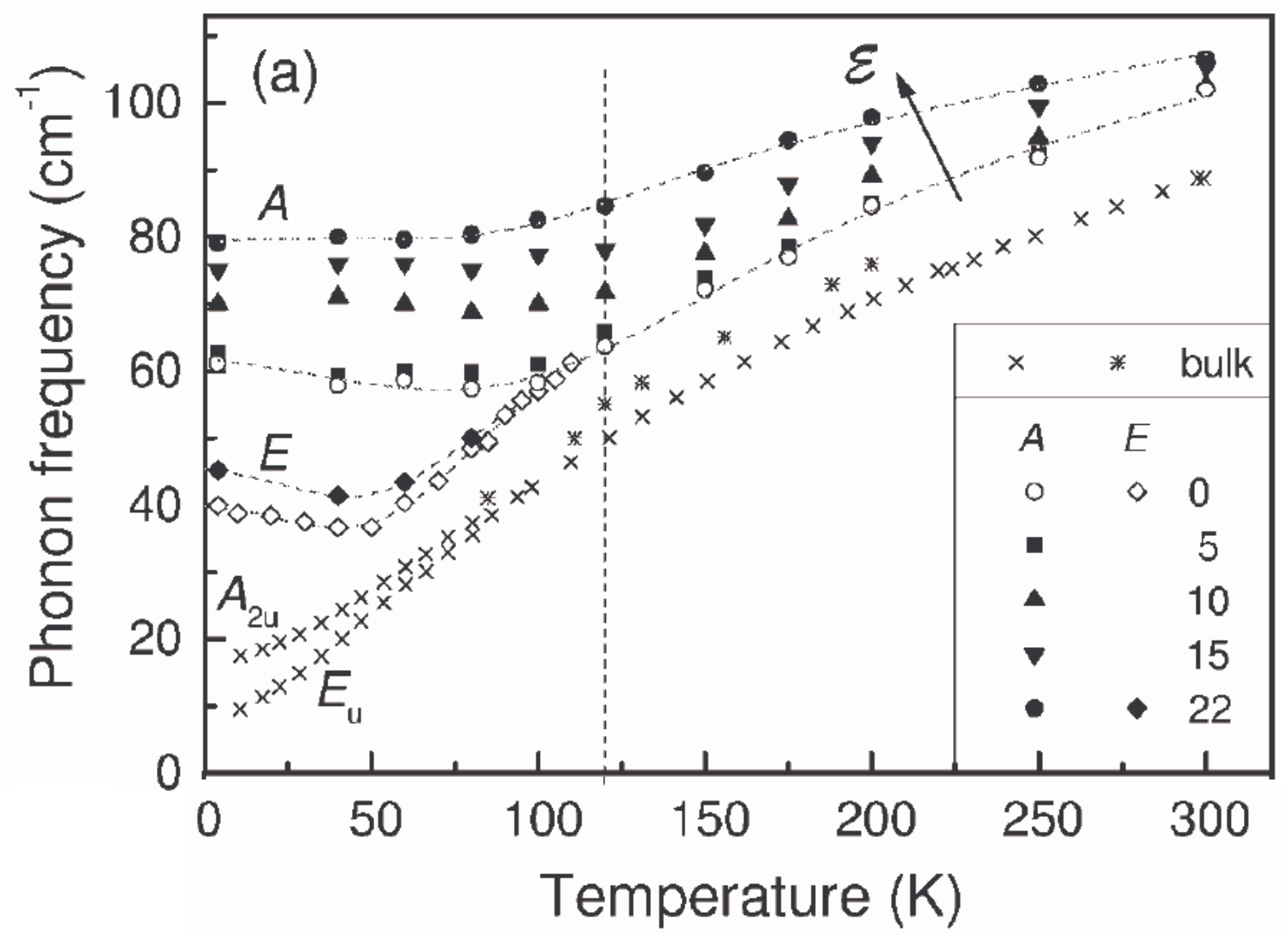

Figure 6

D. A. Tenne and X. X. Xi 


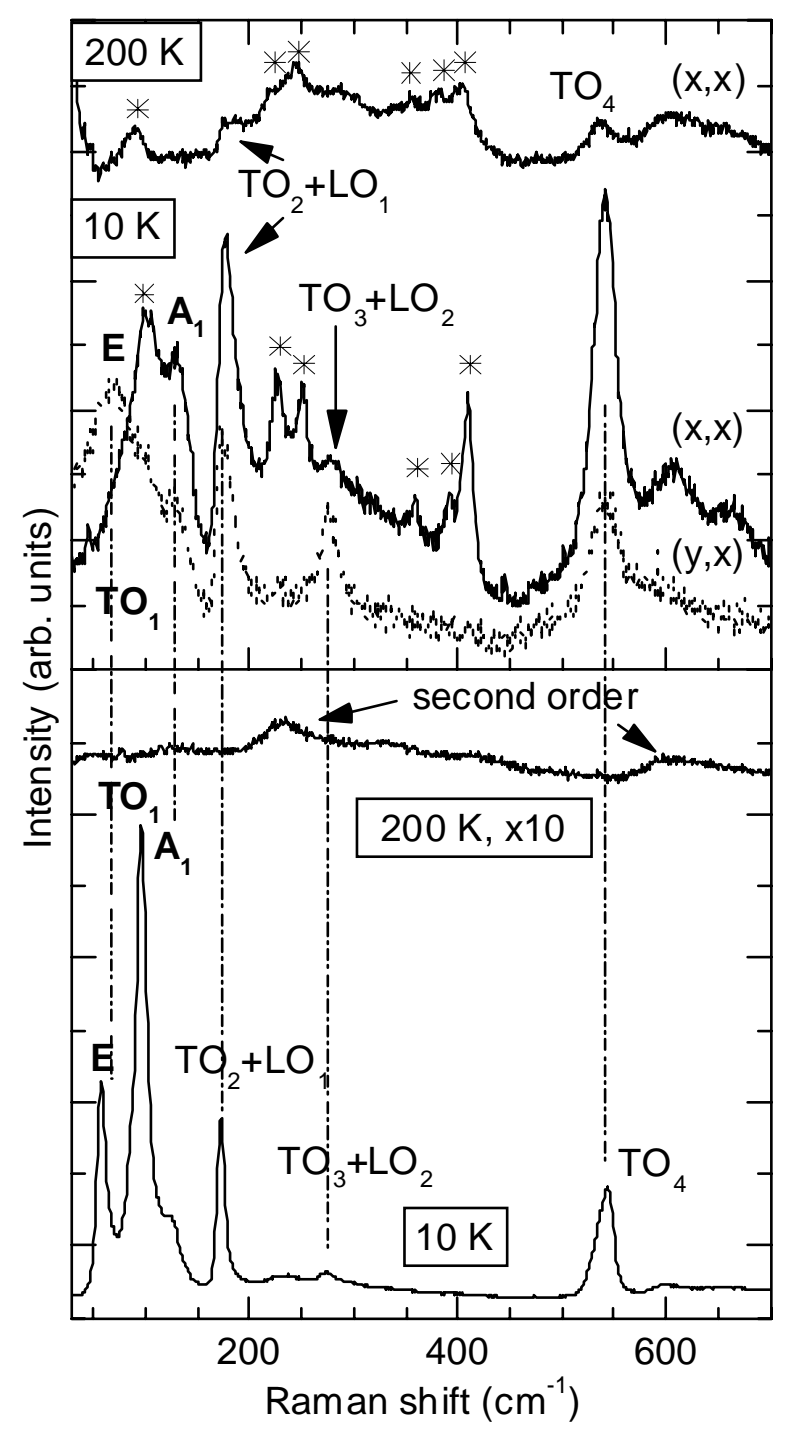

Figure 7

D. A. Tenne and X. X. Xi 


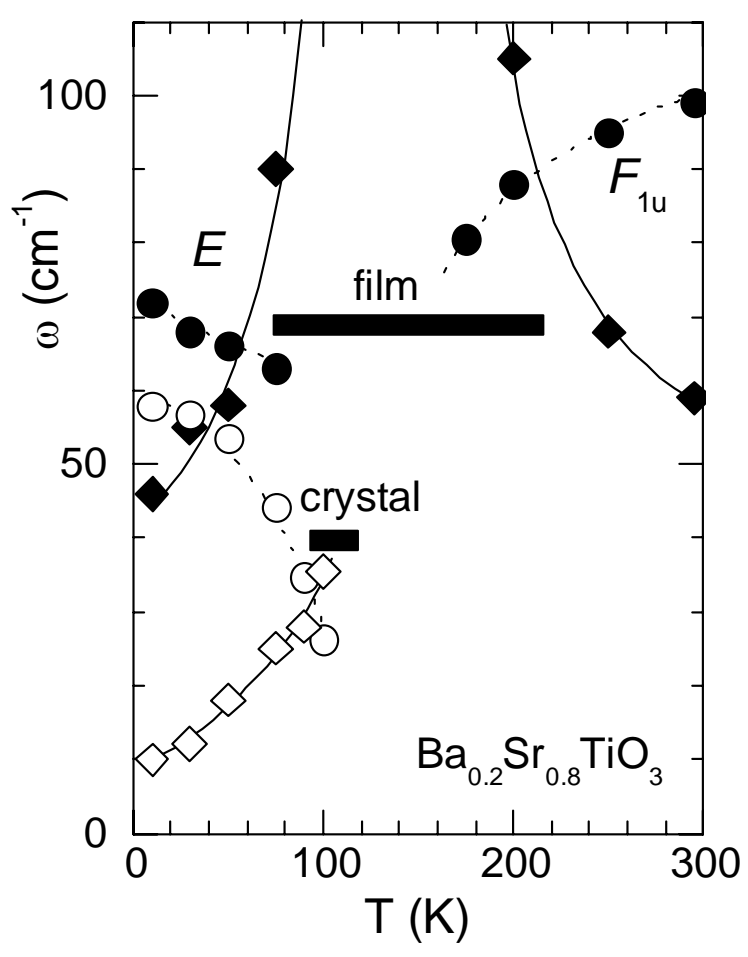

Figure 8

D. A. Tenne and X. X. Xi 




Figure 9

D. A. Tenne and X. X. Xi 




\section{Figure 10 \\ D. A. Tenne and X. X. Xi}




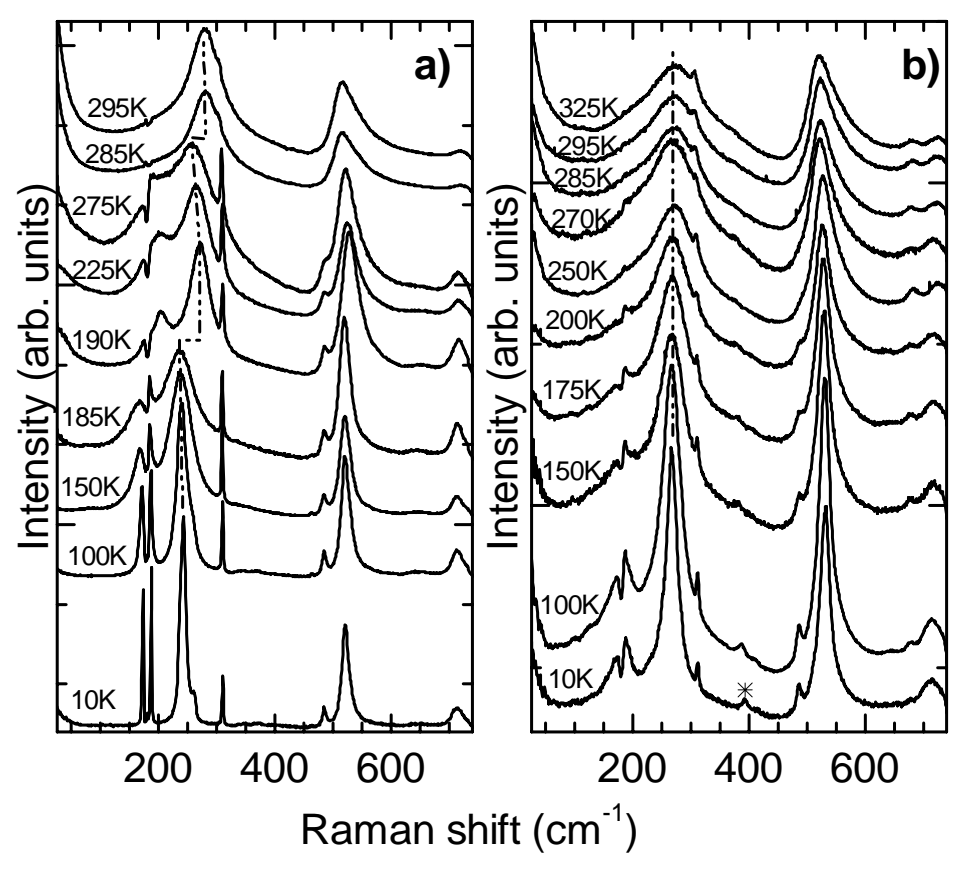

Figure 11

D. A. Tenne and X. X. Xi 


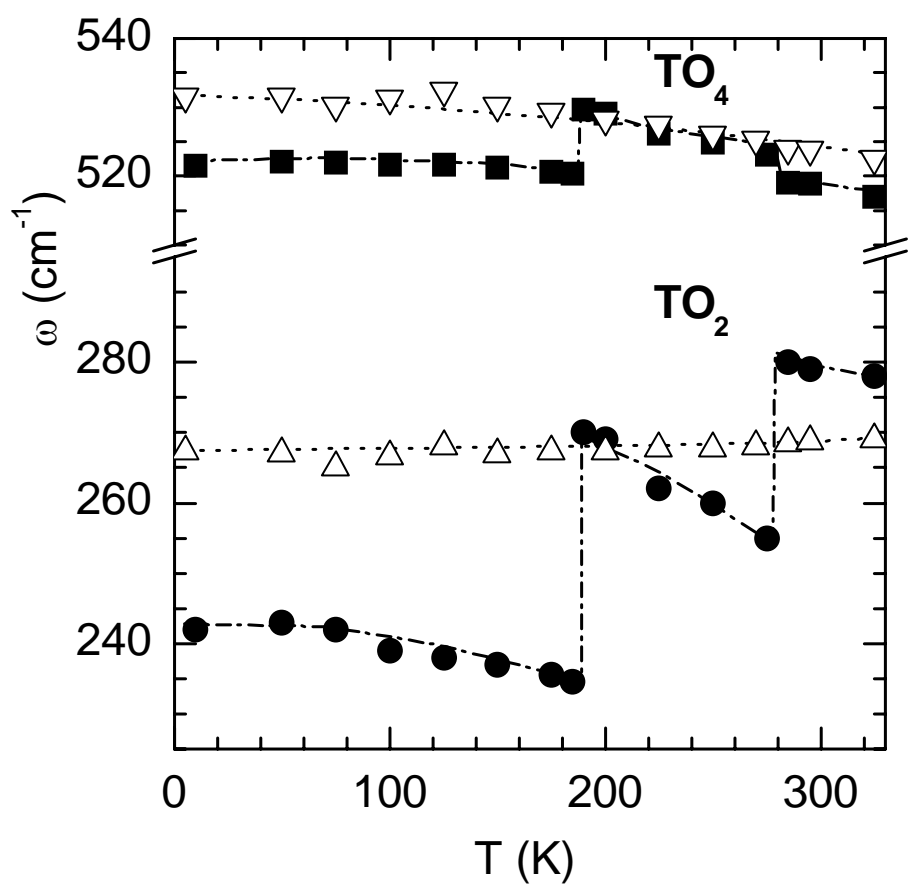

Figure 12

D. A. Tenne and X. X. Xi 
This is an author-produced, peer-reviewed version of this article. The final, definitive version of this document can be found online at Journal of the American Ceramic Society, published by Blackwell Publishing. Copyright restrictions may apply. doi: 10.1111/j.1551-2916.2008.02371.x
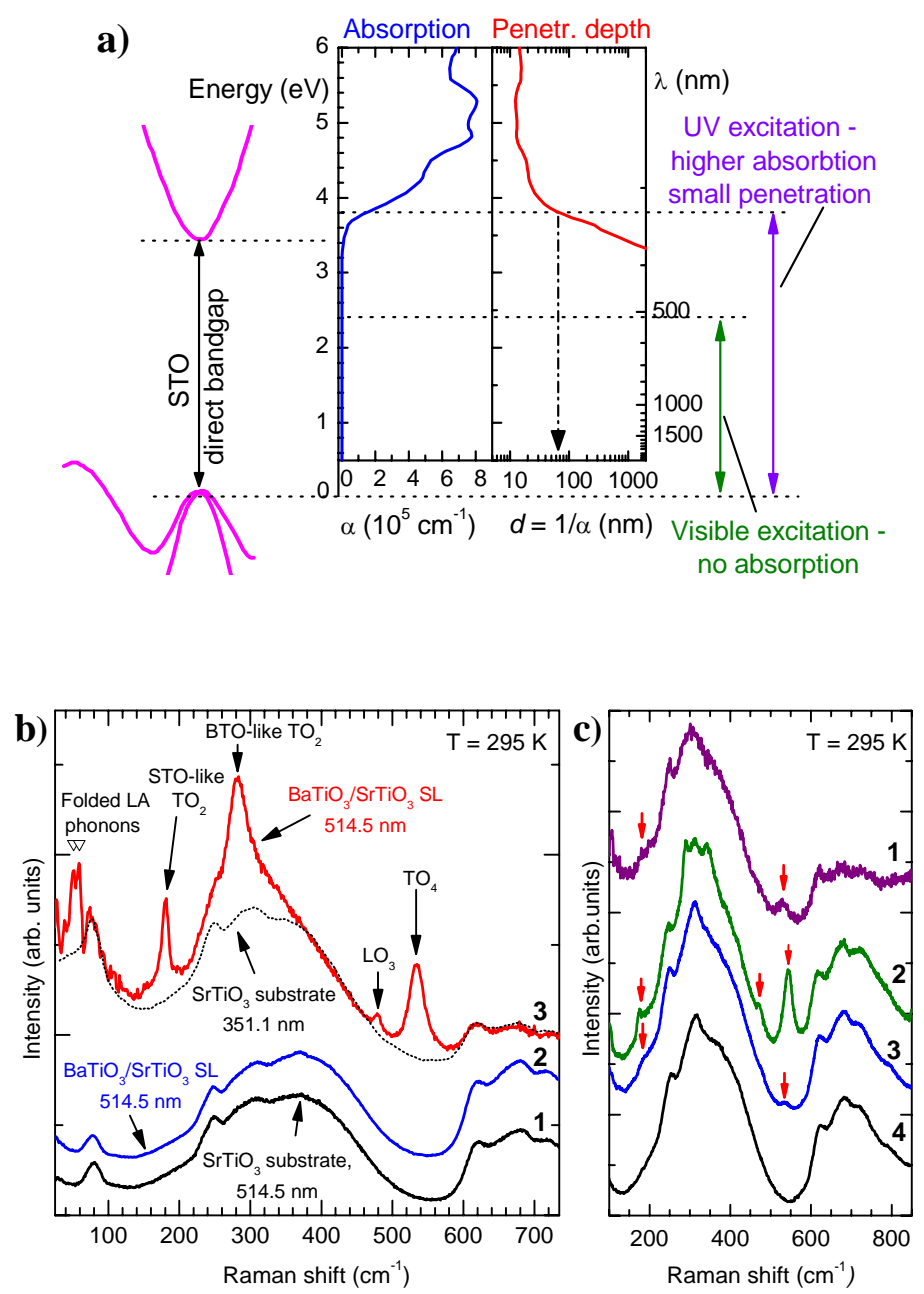

Figure 13

D. A. Tenne and X. X. Xi 




Figure 14

D. A. Tenne and X. X. Xi 




Figure 15

D. A. Tenne and X. X. Xi 

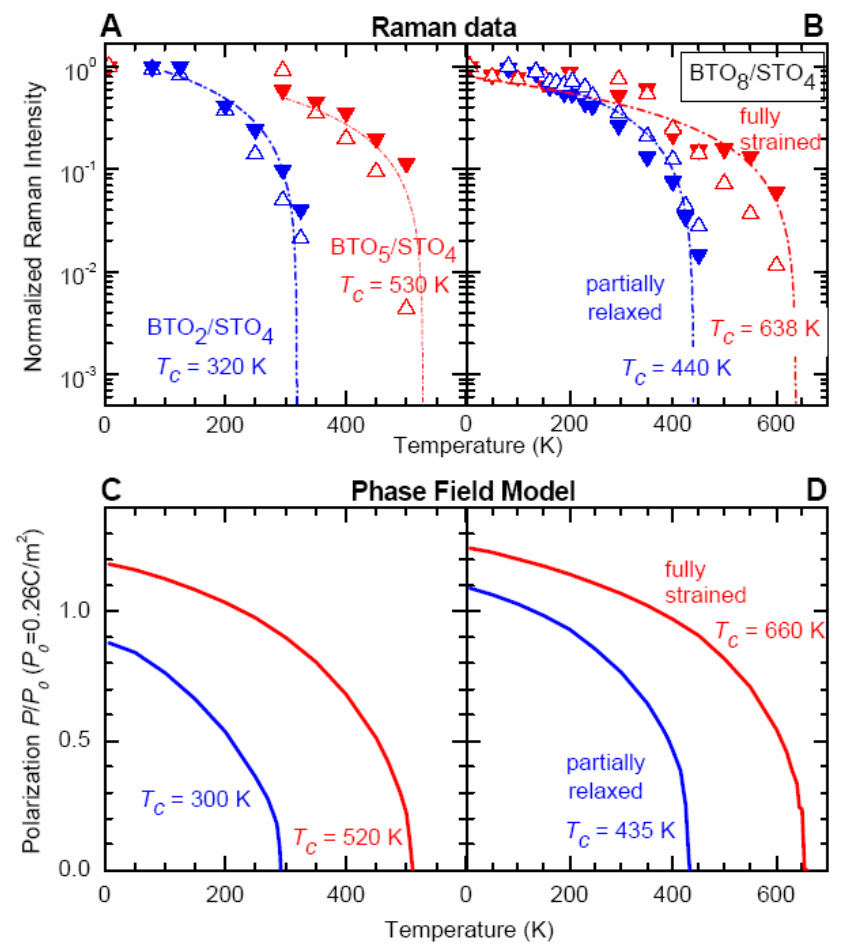

Figure 16

D. A. Tenne and X. X. Xi 


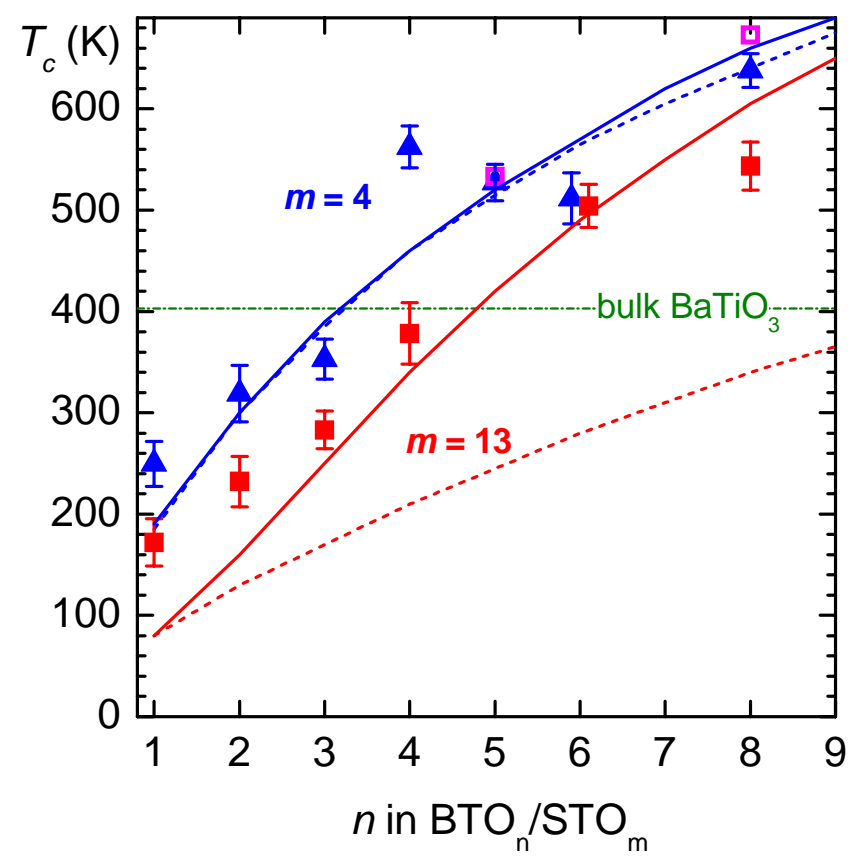

Figure 17

D. A. Tenne and X. X. Xi 